\title{
Vision-based Localization and Attitude Estimation Methods in Natural Environments
}

\section{Bertil Grelsson}


Linköping Studies in Science and Technology

Dissertations, No. 1977

\section{Vision-based Localization and Attitude Estimation Methods in Natural Environments}

Bertil Grelsson

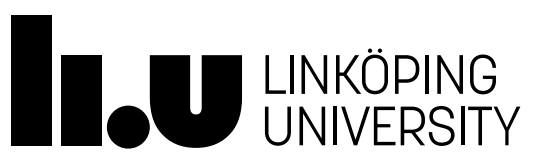

Linköping University

Department of Electrical Engineering

Computer Vision Laboratory

SE-581 83 Linköping, Sweden

Linköping 2019 
Edition 1:1

(C) Bertil Grelsson, 2019

ISBN 978-91-7685-118-0

ISSN 0345-7524

URL http://urn.kb.se/resolve?urn=urn:nbn:se:liu:diva-154159

Published articles have been reprinted with permission from the respective copyright holder.

Typeset using $\mathrm{X}_{4} \mathrm{~T}_{\mathrm{E}} \mathrm{X}$

Printed by LiU-Tryck, Linköping 2019 
For Annika and Hanna 



\section{POPULÄRVETENSKAPLIG SAMMANFATTNING}

Under det senaste decenniet har användningen av obemannade system såsom obemannade flygfarkoster (UAV:er), obemannade ytfartyg (USV:er) och obemannade markfordon (UGV:er) ökat markant och man ser fortsatt en snabb tillväxt. Idag används obemannade system i många vardagliga tillämpningar t.ex. för leveranser i avlägsna områden, för att öka jordbrukets effektivitet och för miljöövervakning till sjöss. Av säkerhetsskäl föredrar man ofta obemannade system vid övervakningsuppdrag i farliga miljöer t.ex. vid detektering av radioaktiv strålning och spaning i katastrofområden efter jordbävningar, orkaner eller under skogsbränder. För säker navigering av de obemannade systemen under dessa uppdrag krävs kontinuerlig och noggrann skattning av deras globala position och orientering.

Under årens lopp har många bildbaserade metoder för positionsskattning utvecklats och då främst för stadsområden. Denna avhandling är främst inriktad mot bildbaserade metoder för noggrann skattning av position och orientering i naturliga miljöer (dvs utanför stadsområden). Bildbaserade metoder har flera egenskaper som gör dem lämpliga som sensorer för global skattning av position och orientering. För det första kan bildsensorer tillverkas och skräddarsys för de flesta applikationer med obemannade system. För det andra kan georefererade terrängmodeller genereras över hela världen från satellitbilder och modellerna kan lagras ombord på farkosterna. I naturliga miljöer finns generellt sett väldigt få georefererade bilder tillgängliga och registrering av bildinformation med terrängmodeller blir därmed det naturliga valet för att skatta position och orientering. Detta är problemområdet som jag inriktat mig mot i de olika bidragen i avhandlingen.

Det första bidraget är en metod för skattning av en global sex frihetsgraders-pose från flygfoton. Först beräknas en lokal höjdkarta med hjälp av struktur-från-rörelse. Den globala posen erhålls ur 3D-transformationen mellan den lokala höjdkartan och en digital höjdmodell. Matchning av höjdinformation anses vara mer robust mot årstidsförändringar än särdragsbaserad matchning.

Det andra bidraget är en metod för noggrann skattning av attityden (tipp- och rollvinkel) genom horisontdetektering. Den är en av ett fåtal metoder som använder en fisheye-kamera för horisontdetektering i flygbilder. Metoden baseras på kantdetektering och ett probabilistiskt röstningsförfarande. Metoden möjliggör att a priori-kunskap om attitydvinklarna kan nyttjas för att göra de initiala attitydskattningarna mer robusta. Skattningarna förfinas därefter genom registrering mot den geometriska horisontlinjen från en digital höjdmodell. Vad vi känner till så är detta den första metoden som tar hänsyn till ljusets brytning i atmosfären vilket möjliggör de väldigt noggranna attitydskattningarna.

Det tredje bidraget är en metod för positionsskattning som baseras på horisontdetektering $\mathrm{i}$ en panoramabild runt ett ytfartyg. Två faltningsnätverk (CNN) tränas för att skatta kamerans orientering samt för att segmentera horisontlinjen i bilden. Ett inlärt korrelationsfilter, som normalt används vid följning av visuella objekt, har anpassats till horisontregistrering med geometriska data från en digital höjdmodell. Omfattande fältförsök som genomförts ute i skärgården påvisar att metoden har en positionsnoggrannhet liknande GPS och att den kan tränas på bilder från ett område och sedan appliceras på bilder från ett annat, tidigare obesökt område.

Faltningsnätverken i det tredje bidraget använder sig som brukligt är av byggstenarna faltningar, aktiveringsfunktioner och pooling. Det fjärde bidraget är inriktat mot aktiveringar och föreslår en ny formulering för att finjustera och optimera en stegvis linjär aktiveringsfunktion under träning av ett CNN. Experiment som gav förbättrade klassificeringsresultat vid finjustering av aktiveringsfunktionen ledde till introduktion av en ny aktiveringsfunktion kallad Shifted Exponential Linear Unit (ShELU). 


\begin{abstract}
Over the last decade, the usage of unmanned systems such as Unmanned Aerial Vehicles (UAVs), Unmanned Surface Vessels (USVs) and Unmanned Ground Vehicles (UGVs) has increased drastically, and there is still a rapid growth. Today, unmanned systems are being deployed in many daily operations, e.g. for deliveries in remote areas, to increase efficiency of agriculture, and for environmental monitoring at sea. For safety reasons, unmanned systems are often the preferred choice for surveillance missions in hazardous environments, e.g. for detection of nuclear radiation, and in disaster areas after earthquakes, hurricanes, or during forest fires. For safe navigation of the unmanned systems during their missions, continuous and accurate global localization and attitude estimation is mandatory.
\end{abstract}

Over the years, many vision-based methods for position estimation have been developed, primarily for urban areas. In contrast, this thesis is mainly focused on vision-based methods for accurate position and attitude estimates in natural environments, i.e. beyond the urban areas. Vision-based methods possess several characteristics that make them appealing as global position and attitude sensors. First, vision sensors can be realized and tailored for most unmanned vehicle applications. Second, geo-referenced terrain models can be generated worldwide from satellite imagery and can be stored onboard the vehicles. In natural environments, where the availability of geo-referenced images in general is low, registration of image information with terrain models is the natural choice for position and attitude estimation. This is the problem area that I addressed in the contributions of this thesis.

The first contribution is a method for full $6 \mathrm{DoF}$ (degrees of freedom) pose estimation from aerial images. A dense local height map is computed using structure from motion. The global pose is inferred from the 3D similarity transform between the local height map and a digital elevation model. Aligning height information is assumed to be more robust to season variations than feature-based matching.

The second contribution is a method for accurate attitude (pitch and roll angle) estimation via horizon detection. It is one of only a few methods that use an omnidirectional (fisheye) camera for horizon detection in aerial images. The method is based on edge detection and a probabilistic Hough voting scheme. The method allows prior knowledge of the attitude angles to be exploited to make the initial attitude estimates more robust. The estimates are then refined through registration with the geometrically expected horizon line from a digital elevation model. To the best of our knowledge, it is the first method where the ray refraction in the atmosphere is taken into account, which enables the highly accurate attitude estimates.

The third contribution is a method for position estimation based on horizon detection in an omnidirectional panoramic image around a surface vessel. Two convolutional neural networks (CNNs) are designed and trained to estimate the camera orientation and to segment the horizon line in the image. The MOSSE correlation filter, normally used in visual object tracking, is adapted to horizon line registration with geometric data from a digital elevation model. Comprehensive field trials conducted in the archipelago demonstrate the GPS-level accuracy of the method, and that the method can be trained on images from one region and then applied to images from a previously unvisited test area.

The CNNs in the third contribution apply the typical scheme of convolutions, activations, and pooling. The fourth contribution focuses on the activations and suggests a new formulation to tune and optimize a piecewise linear activation function during training of CNNs. Improved classification results from experiments when tuning the activation function led to the introduction of a new activation function, the Shifted Exponential Linear Unit (ShELU). 


\section{Acknowledgments}

Today, it seems like ages ago, that day, when I was asked by my employer if I would be interested in a position as an industrial $\mathrm{PhD}$ student. I recall that my biggest concern at the time was what it would feel like going back to school again after all those years. After all, at that time, I had spent almost twenty years working in industry. Anyway, I did not have to ponder for long. My desire to learn something new in a completely new environment was much stronger than any doubts I had. I accepted the offer and challenge, and today I am very glad I took this route. Mostly, it has been a joyful and very rewarding journey. My great Thanks to all former and current members of the Computer Vision Laboratory for providing an inspiring and friendly working environment over the years. I can just note that being a PhD student at CVL is very, very different from a regular working day at SAAB. And I fully agree with former president Obama's slogan, "Change We Need" - for inspiration, motivation, and to develop as a person.

Some of the people at CVL and SAAB have influenced my work and my writing of this thesis more than others. Special thanks to:

- My supervisor Michael Felsberg for excellent guidance and support, and being a true source of inspiration, always perceiving new opportunities and hurdling any obstacles in our joint research challenges.

- My co-supervisor Per-Erik Forssén for interesting and fruitful discussions on my research topic and for giving me valuable insights how to compose and write scientific papers.

- The CIMSMAP project group comprising Michael Felsberg, Per-Erik Forssén, Leif Haglund, Folke Isaksson, Sören Molander and Pelle Carlbom for providing great technical support and advice, and at each meeting generating a diversity of conceivable research paths, some of them leading to this thesis, some of them still being unexplored.

- Andreas Robinson for all your efforts when we jointly worked on two papers, for setting up my working environment and for keeping my computers happy. 
- Leif Haglund and his colleagues at Vricon who throughout my studies have supported me with high-class reference data.

- The Saab Kockums team in Karlskrona who gladly supported me conducting the field trials with the Piraya.

- Hannes Ovrén for kindly sharing his thesis manuscript and chapter styles, which saved me lots of valuable time writing this thesis.

I would also like to thank my family, colleagues and friends for their everyday life support, most notably:

- Annika and Hanna for your love, patience and understanding during this period. Conducting doctoral research takes time. During some periods, it may take lots of time. I truly appreciate all the ground support and cheering you have provided these years and for still supporting me when I chose to spend yet another day at LiU.

- Thanks also to my employer Saab Dynamics for giving me the opportunity to undertake the studies leading to this thesis. I am confident that it will turn out a mutual win-win situation over time.

This work was funded by the Swedish Governmental Agency for Innovation Systems, VINNOVA, under contracts NFFP5 2010-01249 and 2013-05243.

This work was partially supported by the Wallenberg AI, Autonomous Systems and Software Program (WASP) funded by the Knut and Alice Wallenberg Foundation.

Linköping, March 2019

Bertil Grelsson

\section{About the cover}

The front cover shows one image captured with a Ladybug camera onboard the Piraya USV in the Västervik archipelago. The red line is the horizon profile detected by the method in Paper F. The back cover shows one fisheye image captured in an aerial flight trial conducted in the vicinity of Linköping. The red ellipse is the horizon line detected by the method in Paper C. 


\section{Contents}

Abstract v v

Acknowledgments viii

$\begin{array}{lll}\text { Contents } & \text { ix }\end{array}$

I Background 1

1 Introduction $\quad 3$

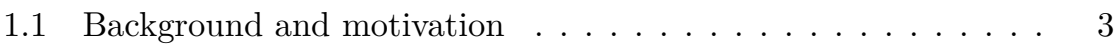

1.2 Goal of this thesis . . . . . . . . . . . . . . 5

1.3 Outline ..................... . . 7

1.4 Included publications . . . . . . . . . . . . . . . . . . . . . . 9

1.5 Other publications . . . . . . . . . . . . . 15

2 Taxonomy of vision-based pose estimation approaches $\quad 17$

2.1 Image-based methods . . . . . . . . . . . . . . . . . . . . . 18

2.2 SLAM methods . . . . . . . . . . . . . . . . . 18

2.3 Multiple modality data methods . . . . . . . . . . . . . . 19

3 Camera Models 2

3.1 Pinhole camera model . . . . . . . . . . . . . . . . . 21

3.2 Lens distortion . . . . . . . . . . . . . . . . . 23

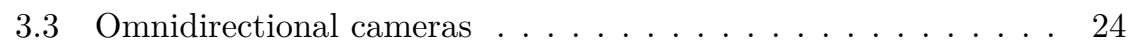

3.4 Camera calibration . . . . . . . . . . . . . . . . 27

4 Multiple-view geometry 29

4.1 Epipolar geometry . . . . . . . . . . . . . . . . . . . . . . 30

4.2 Local pose estimation . . . . . . . . . . . . . . . . 31

4.3 Structure from Motion . . . . . . . . . . . . . . . . . . . . . . . . . . 32

4.4 Dense 3D reconstruction . . . . . . . . . . . . 32

5 Geometric geographic information $\quad 35$

5.1 Digital Elevation Models . . . . . . . . . . . . . . . 35 
5.2 Vision-based 3D models . . . . . . . . . . . . . . . 36

5.3 Geometric horizon . . . . . . . . . . . . . . 36

6 Horizon detection $\quad 41$

6.1 Hough transform . . . . . . . . . . . . . . . . . . . . . 42

6.2 Hough voting - considerations for real images . . . . . . . . . . . . 46

6.3 Extraction of horizon edge pixels . . . . . . . . . . . 46

7 Convolutional Neural Networks $\quad 49$

7.1 Common layer types . . . . . . . . . . . . . . . . . . . . . . 49

7.2 Nonlinear activation functions . . . . . . . . . . . . . . . . . . . 50

7.3 Transfer learning . . . . . . . . . . . . . . . . . 54

8 Registration methods $\quad \mathbf{5 7}$

$8.13 \mathrm{D}-3 \mathrm{D}$ registration $\ldots \ldots \ldots \ldots \ldots$

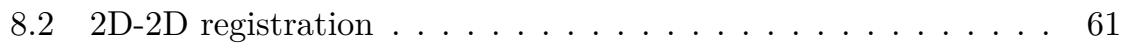

$8.31 \mathrm{D}-1 \mathrm{D}$ registration $\ldots \ldots \ldots \ldots . \ldots \ldots$

9 Evaluation $\quad \mathbf{6 5}$

9.1 Ground truth generation . . . . . . . . . . . . . . 65

9.2 Evaluation measures . . . . . . . . . . . . . . . . . . 66

9.3 Evaluation analysis of onboard operational use . . . . . . . . 69

10 Concluding remarks $\quad 71$

10.1 Conclusions of results . . . . . . . . . . . . . 71

10.2 Future work . . . . . . . . . . . . . . . . . . . . . . . . . . . . . . .

10.3 Impact on society . . . . . . . . . . . . . . . 74

$\begin{array}{lr}\text { Bibliography } & \mathbf{7 5}\end{array}$

$\begin{array}{lr}\text { II Publications } & 81\end{array}$

$\begin{array}{lll}\text { Paper A Efficient 7D Aerial Pose Estimation } & 83\end{array}$

$\begin{array}{lll} & \text { Probabilistic Hough voting for attitude estimation } & 93\end{array}$

$\begin{array}{lll}\text { Paper C Highly Accurate Attitude Estimation via Horizon } & 107\end{array}$

Improved Learning in Convolutional Neural Net-

Paper D works with Shifted Exponential Linear Units 137 (ShELUs)

Paper E HorizonNet for visual terrain navigation

Paper F $\begin{aligned} & \text { GPS-level Accurate Camera Localization with Hori- } \\ & \text { zonNet }\end{aligned}$ 
PART I

BACKGROUND 



\section{INTRODUCTION}

\subsection{Background and motivation}

In this thesis, I have addressed vision-based methods for localization and attitude estimation of vehicle-mounted cameras in natural environments. The methods are primarily intended for operation onboard unmanned systems, such as Unmanned Aerial Vehicles (UAVs), Unmanned Surface Vessels (USVs) and Unmanned Ground Vehicles (UGVs), see figure 1.1. The group of unmanned systems are commonly referred to as UxVs. The usage of UxVs has increased drastically over the last decade, and there is still a rapid growth. Today, unmanned systems are employed to operate missions in a large variety of environments. Some examples are: UAVs carry out aerial deliveries of medical products to hospitals in remote areas [63], USVs perform environmental monitoring, patrolling, and search and rescue missions in maritime areas [44], UGVs are utilized for precision spraying and crop harvesting to increase efficiency of agriculture [6]. Furthermore, unmanned systems are often the preferred choice for surveillance missions in hazardous environments, e.g. for detection of nuclear radiation [18], and in disaster areas after earthquakes, hurricanes or during forest fires [61].
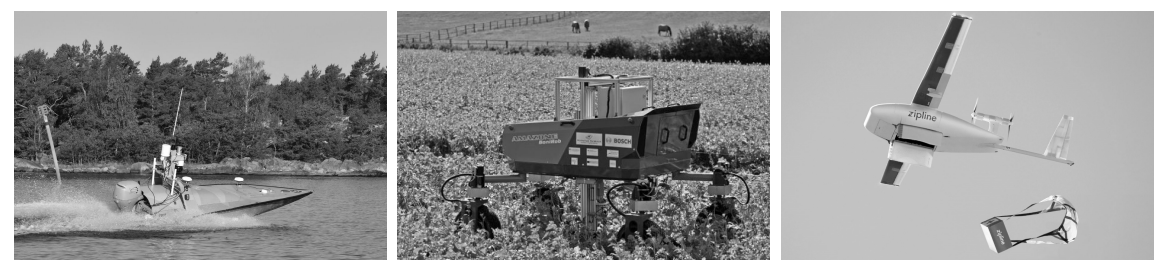

Figure 1.1: Example images of USV (left), UGV (middle) - courtesy of Deepfield Robotics, and UAV (right) - courtesy of Zipline. 
To perform their missions, the UxVs are controlled either autonomously by computers onboard the vehicle or tele-operated by a pilot. For safe navigation of the UxVs, continuous and accurate global pose (position and orientation) estimation is mandatory. In many applications today, the UxVs are fully reliant on accurate position measurements provided by the Global Positioning System (GPS), or another Global Navigation Satellite System (GNSS), as a single source position sensor. However, the GPS signal is not always available and reliable. GPS outages are rare [59], but they do occur and they need to be accounted for in the vehicle navigation system. Perhaps a more severe issue, in the field trials conducted to evaluate two of the methods developed in this thesis, we experienced a case with a short time period of completely erroneous GPS measurements. The GPS receiver repeatedly computed position estimates which were located south of the equator, and not in southern Sweden where the trials were actually performed. The GPS receiver fed the vehicle autopilot with these bad position estimates, and the autopilot completely lost control of its true position and the appropriate heading to proceed to the next waypoint in the planned mission. The pilot, being standby to tele-operate the vehicle, had to save the situation. An autonomous system must be capable of handling such erroneous position estimates from the GPS. Furthermore, it is well known that the position accuracy of the GPS may be degraded due to multi-path effects, especially in high-rise environments. Also, in hostile scenarios, the GPS signal may be jammed or spoofed leading to no or erroneous measurements. All in all, the GPS is a great position sensor when available and reliable. But in many cases, there is a need for a complementary and independent position sensor besides the GPS for safe navigation of the UxVs.

Vision sensors, or cameras, are ubiquitous in today's society. Cameras can be realized in a large variety of sizes, image resolution and field of view (FOV). Their physical characteristics can be adapted and tailored for most $\mathrm{UxV}$ applications. Today, large databases of geo-tagged images can be made available in most urban areas over the world. Satellites can provide radar and hyperspectral image data to generate geo-referenced terrain models worldwide [42], [55]. The geo-referenced databases of the operational area may be stored onboard the UxVs. Online registration of information from images captured onboard the $\mathrm{UxVs}$ with the databases enables accurate and global pose estimates to be provided during the missions. These characteristics make vision-based methods appealing as a pose estimation sensor onboard the UxVs.

Over the years, a large number of vision-based methods for localization and attitude estimation have been proposed. But the combinations of $\mathrm{UxV}$ type (aerial, land, sea), terrain type (urban, rural, mountains, forest, desert, farmland), season (summer, winter, with and without snow), lighting conditions (day, night, sunshine, clouds, fog), FOV (normal, narrow, omnidirectional) are numerous. Most pose estimation methods can only handle a few of these combinations and there are still open research areas within the field. Natural environments (beyond urban areas) where there are relatively few images 
available, in combination with omnidirectional cameras is still a relatively unexplored field. This is the research area that has been addressed in this thesis.

\section{Definitions}

In this thesis, the following terminology has been used:

- Pose estimation refers to an estimate of the position and the orientation (rotation angles) of the camera or the vehicle, see figure 1.2.

- Localization refers to an estimate of the position.

- Attitude estimation refers to an estimate of the orientation.

- Navigation refers to the process of modelling, measuring, and estimating the movement of a vehicle over time, from one place to another.

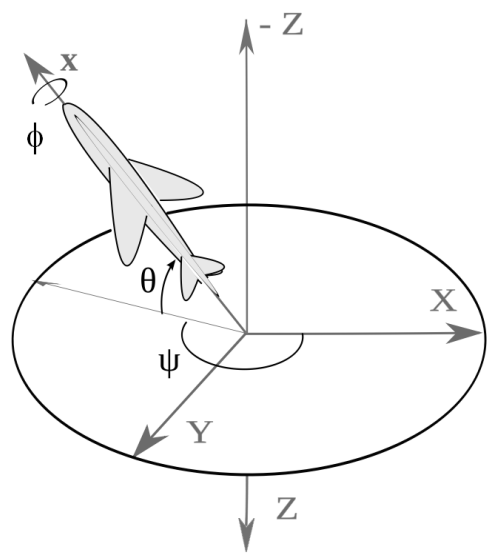

Figure 1.2: Definition of the vehicle 6 DoF pose in a world coordinate frame. $\mathrm{X}=$ north, $\mathrm{Y}=$ east, $\mathrm{Z}=$ down, $\psi=$ yaw, $\theta=$ pitch, $\phi=$ roll.

\subsection{Goal of this thesis}

The work leading to this thesis has been conducted in two projects within the framework of two large Swedish research programs called NFFP5 (Nationellt Flygtekniskt ForskningsProgram fas 5 - National Aviation Engineering Research Programme phase 5) and WASP (Wallenberg AI, Autonomous Systems and Software Program). The work in the two projects was performed separated in time. The former project was carried out 2011-2014, and the latter project was conducted 2016-2019. 
The project within NFFP5 was named CIMSMAP "Correlation of image sequences with 3D mapping data for positioning in airborne applications". The goal of the project was to develop automated vision-based methods for global pose (position and orientation) estimation of aerial vehicles. The main idea was to achieve global pose estimation via registration of aerial images with a geo-referenced 3D model generated from aerial images captured in a previous instance in time. The results from the CIMSMAP project were the basis for my licentiate thesis [23].

The time between the ending of the first project and the start of the second project was not more than two years. But during this time, there had been a paradigm shift within the computer vision community. The first project was mainly conducted in the "Least-squares era", whereas when we applied for funding from the WASP foundation for the second project, the community had entered the "Deep-learning era". The main interest of the WASP program is to raise the general competence of autonomous systems within academia and industry in Sweden. The goal of my WASP project was to develop visionbased pose estimation methods to aid navigation of autonomous vehicles. The additional aim was to explore how deep learning methods could be utilized within this area of research.

The conduction of the research work within the two projects shows great similarity. In CIMSMAP, the developed methods for global pose estimation were evaluated using true aerial imagery captured in flight trials with manned aircraft. Figure 1.3 shows the experimental aircraft used in one of the field trials, and an example fisheye image from the trial illustrates the detected horizon line utilized for registration with a digital elevation model.
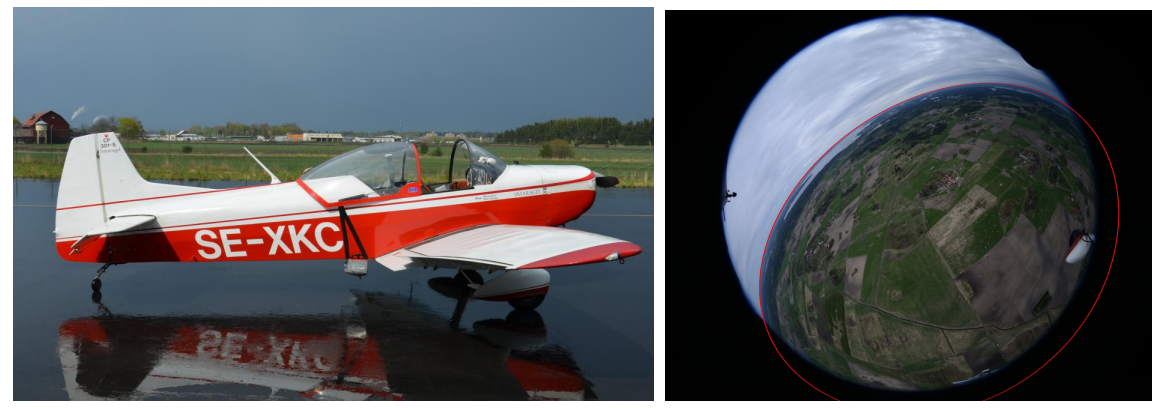

Figure 1.3: Test aircraft carrying the fisheye camera (left). Detected horizon line in fisheye image (right). Images from Paper C.

The WASP program also gave us PhD students the opportunity to capture real world images from remotely operated vehicles to develop and evaluate our proposed methods. Figure 1.4 shows the Piraya USV, from Saab Kockums, carrying our omnidirectional camera, and an example image with the detected 
horizon line in the panoramic image used for registration with a digital elevation model. The field trials with the Piraya were conducted as part of a larger demonstration together with some of my fellow WASP students. A news report from the trials with video illustrations can be found in [57].
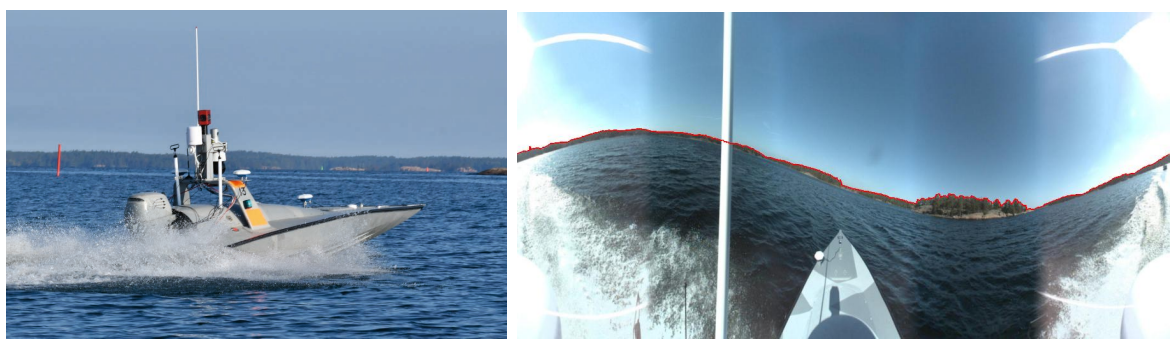

Figure 1.4: Piraya USV used in the field trial (left). Segmented horizon line in the Ladybug panoramic image (right). Images from Paper F.

Looking back, I would like to express my great appreciation to these two research programs having had the opportunity to be involved with the planning and conduction of field trials intended to evaluate the research methods you have designed and proposed yourself. To me, there is no better moment in research than when your proposed method or theory can be proven to work on true real world data captured in trials you have participated in yourself.

\subsection{Outline}

This thesis consists of two main parts. The first part presents the background theory for the vision-based global pose estimation methods. The second part contains six publications related to this topic. Parts of the material presented in this thesis also appeared in my licentiate thesis [23].

The background theory is divided into the following chapters:

- Chapter 2: Taxonomy of vision-based pose estimation approaches gives an overview of the main concepts of vision-based pose estimation methods and classify the methods based on the type of reference data used for registration.

- Chapter 3: Camera models describes the mathematical models employed for the image projection types used in this thesis.

- Chapter 4: Multiple-view geometry presents the basics of epipolar geometry and the principle behind 3D reconstructions to generate digital elevation models. 
- Chapter 5: Geometric geographic information describes different types of digital elevation models and how to compute the geometric horizon from DEM data.

- Chapter 6: Horizon detection illustrates how Hough voting can be employed to detect and extract the horizon line in omnidirectional images.

- Chapter 7: Convolutional neural networks describes how the nonlinear activation function could be locally tuned in a CNN, and how the concept of transfer learning was used for horizon line detection.

- Chapter 8: Registration methods presents the methods used in this thesis for registration of image information with digital elevation model data.

- Chapter 9: Evaluation metrics discusses the most common metrics for evaluation of the pose estimation methods.

- Chapter 10: Concluding remarks summarizes the work in this thesis and looks towards the future. 


\title{
1.4 Included publications
}

\section{Paper A: "Efficient 7D aerial pose estimation"}

B. Grelsson, M. Felsberg, and F. Isaksson. "Efficient 7D aerial pose estimation". In: Robot Vision (WORV), 2013 IEEE Workshop on. IEEE. 2013, pp. 88-95

\begin{abstract}
A method for online global pose estimation of aerial images by alignment with a georeferenced 3D model is presented. Motion stereo is used to reconstruct a dense local height patch from an image pair. The global pose is inferred from the 3D transform between the local height patch and the model. For efficiency, the sought 3D similarity transform is found by leastsquares minimizations of three $2 \mathrm{D}$ subproblems. The method does not require any landmarks or reference points in the 3D model, but an approximate initialization of the global pose, in our case provided by onboard navigation sensors, is assumed. Real aerial images from helicopter and aircraft flights are used to evaluate the method. The results show that the accuracy of the position and orientation estimates is significantly improved compared to the initialization and our method is more robust than competing methods on similar datasets. The proposed matching error computed between the transformed patch and the map clearly indicates whether a reliable pose estimate has been obtained.
\end{abstract}

Contributions: In this paper, a local height map of an urban area below the aircraft is computed using motion stereo. The global pose of the aircraft is inferred from the 3D similarity transform between the local height map and a geo-referenced 3D model of the area. The main novelty of the paper is a framework that enables the $3 \mathrm{D}$ similarity transform to be reliably and robustly estimated by solving three $2 \mathrm{D}$ subproblems.

The author contributed to the design of the method, implemented the algorithms, performed the evaluation and wrote the main part of the manuscript. 


\title{
Paper B: "Probabilistic Hough voting for attitude estimation from aerial fisheye images"
}

B. Grelsson and M. Felsberg. "Probabilistic Hough voting for attitude estimation from aerial fisheye images". In: Scandinavian Conference on Image Analysis. Springer. 2013, pp. 478-488

\begin{abstract}
For navigation of unmanned aerial vehicles (UAVs), attitude estimation is essential. We present a method for attitude estimation (pitch and roll angle) from aerial fisheye images through horizon detection. The method is based on edge detection and a probabilistic Hough voting scheme. In a flight scenario, there is often some prior knowledge of the vehicle altitude and attitude. We exploit this prior to make the attitude estimation more robust by letting the edge pixel votes be weighted based on the probability distributions for the altitude and pitch and roll angles. The method does not require any sky/ground segmentation as most horizon detection methods do. Our method has been evaluated on aerial fisheye images from the internet. The horizon is robustly detected in all tested images. The deviation in the attitude estimate between our automated horizon detection and a manual detection is less than $1^{\circ}$.
\end{abstract}

Contributions: This paper introduces one of only a few available methods using omnidirectional aerial images for absolute attitude estimation from horizon detection. The main novelty is the combination of (1) computing attitude votes from projection of edge pixels and their orientation on the unit sphere, and (2) weighting the votes based on the prior probability distributions of the altitude and pitch and roll angles, in order to obtain a robust and geometrically sound attitude estimate.

The author contributed to the idea and design of the method, implemented the algorithms, conducted the evaluation and wrote the main part of the manuscript. 


\title{
Paper C: "Highly accurate attitude estimation via horizon detec- tion"
}

B. Grelsson, M. Felsberg, and F. Isaksson. "Highly accurate attitude estimation via horizon detection". In: Journal of Field Robotics 33.7 (2016), pp. 967-993

\begin{abstract}
Attitude (pitch and roll angle) estimation from visual information is necessary for GPS free navigation of airborne vehicles. We propose a highly accurate method to estimate the attitude by horizon detection in fisheye images. A Canny edge detector and a probabilistic Hough voting scheme are used to compute an approximate attitude and the corresponding horizon line in the image. Horizon edge pixels are extracted in a band close to the approximate horizon line. The attitude estimates are refined through registration of the extracted edge pixels with the geometrical horizon from a digital elevation map (DEM), in our case the SRTM3 database. The proposed method has been evaluated using 1629 images from a flight trial with flight altitudes up to $600 \mathrm{~m}$ in an area with ground elevations ranging from sea level up to $500 \mathrm{~m}$. Compared with the ground truth from a filtered IMU/GPS solution, the standard deviation for the pitch and roll angle errors are $0.04^{\circ}$ and $0.05^{\circ}$, respectively, with mean errors smaller than $0.02^{\circ}$. The errors obtained are about one order of magnitude smaller than for any previous vision-based method for attitude estimation from horizon detection in aerial images. To achieve the high accuracy attitude estimates, the ray refraction in the earth atmosphere has been taken into account.
\end{abstract}

Contributions: This paper addresses the problem of attitude estimation from horizon detection in images. The paper presents the very first method where the attitude estimates from the horizon in fisheye images is refined through registration with the geometrically expected horizon from a digital elevation model. It is one of few methods where the ray refraction in the atmosphere is taken into account which contributes to the highly accurate pose estimates.

The author planned and participated in the conduction of the field trials, contributed to the idea and design of the method, implemented the algorithms, performed the evaluation and wrote the main part of the manuscript. 


\title{
Paper D: "Improved Learning in Convolutional Neural Networks with Shifted Exponential Linear Units (ShELUs)"
}

B. Grelsson and M. Felsberg. "Improved Learning in Convolutional Neural Networks with Shifted Exponential Linear Units (ShELUs)". In: 2018 24th International Conference on Pattern Recognition (ICPR). IEEE. 2018, pp. 517-522

\begin{abstract}
The Exponential Linear Unit (ELU) has been proven to speed up learning and improve the classification performance over activation functions such as ReLU and Leaky ReLU for convolutional neural networks. The reasons behind the improved behavior are that ELU reduces the bias shift, it saturates for large negative inputs and it is continuously differentiable. However, it remains open whether ELU has the optimal shape and we address the quest for a superior activation function.

We use a new formulation to tune a piecewise linear activation function during training, to investigate the above question, and learn the shape of the locally optimal activation function. With this tuned activation function, the classification performance is improved and the resulting, learned activation function shows to be ELU-shaped irrespective if it is initialized as a RELU, LReLU or ELU. Interestingly, the learned activation function does not exactly pass through the origin indicating that a shifted ELU-shaped activation function is preferable. This observation leads us to introduce the Shifted Exponential Linear Unit (ShELU) as a new activation function.

Experiments on Cifar-100 show that the classification performance is further improved when using the ShELU activation function in comparison with ELU. The improvement is achieved when learning an individual bias shift for each neuron.
\end{abstract}

Contributions: This paper presents a new formulation to tune and locally optimize a piecewise linear activation function during training of convolutional neural networks. Improved classification results from experiments when tuning the activation function lead to the introduction of a new activation function, the Shifted Exponential Linear Unit (ShELU).

The author contributed to the idea and design of the method, implemented the algorithms, performed the evaluation and wrote the main part of the manuscript. 


\title{
Paper E: "HorizonNet for visual terrain navigation"
}

B. Grelsson, A. Robinson, M. Felsberg, and F. Khan. "HorizonNet for visual terrain navigation". In: 2018 3rd International Conference on Image Processing, Applications and Systems (IPAS). IEEE. 2018

\begin{abstract}
This paper investigates the problem of position estimation of unmanned surface vessels (USVs) operating in coastal areas or in the archipelago. We propose a position estimation method where the horizon line is extracted in a 360 degree panoramic image around the USV. We design a CNN architecture to determine an approximate horizon line in the image and implicitly determine the camera orientation (the pitch and roll angles). The panoramic image is warped to compensate for the camera orientation and to generate an image from an approximately level camera. A second CNN architecture is designed to extract the pixelwise horizon line in the warped image. The extracted horizon line is correlated with digital elevation model (DEM) data in the Fourier domain using a MOSSE correlation filter. Finally, we determine the location of the maximum correlation score over the search area to estimate the position of the USV. Comprehensive experiments are performed in a field trial in the archipelago. Our approach provides promising results by achieving position estimates with GPS-level accuracy.
\end{abstract}

Contributions: The paper proposes a new method for vision-based position estimation based on horizon detection in a $360^{\circ}$ panoramic image. Two CNNs are designed and trained to estimate the camera orientation and to segment the horizon line in the image. The MOSSE correlation filter, normally used in visual object tracking, is adapted to horizon line registration with geometric data from a digital elevation model. Comprehensive field trials conducted in the archipelago demonstrate the GPS-level accuracy of the proposed method.

The author planned and participated in the conduction of the field trials, contributed to the idea and design of the method, implemented the algorithms for the baseline method and for generation of the training data, performed the training and the evaluation, and wrote the main part of the manuscript. 


\title{
Paper F: "GPS-level Accurate Camera Localization with Horizon- Net"
}

B. Grelsson, A. Robinson, M. Felsberg, and F. Khan. "GPS-level Accurate Camera Localization with HorizonNet". In: Submitted to Journal of Field Robotics (2019)

\begin{abstract}
This paper investigates the problem of position estimation of unmanned surface vessels (USVs) operating in coastal areas or in the archipelago. We propose a position estimation method where the horizon line is extracted in a $360^{\circ}$ panoramic image around the USV. We design a CNN architecture to determine an approximate horizon line in the image and implicitly determine the camera orientation (the pitch and roll angles). The panoramic image is warped to compensate for the camera orientation and to generate an image from an approximately level camera. A second CNN architecture is designed to extract the pixelwise horizon line in the warped image. The extracted horizon line is correlated with digital elevation model (DEM) data in the Fourier domain using a MOSSE correlation filter. Finally, we determine the location of the maximum correlation score over the search area to estimate the position of the USV. Comprehensive experiments are performed in field trials conducted over three days in the archipelago. Our approach provides excellent results by achieving robust position estimates with GPS-level accuracy in previously unvisited test areas.
\end{abstract}

Contributions: In this paper, a method is proposed for vision-based position estimation based on horizon detection in a $360^{\circ}$ panoramic image. Comprehensive field trials performed over three days in different locations of the Swedish east-coast archipelago demonstrate that: (1) our method can be trained on previously captured image data from one region and achieve GPSlevel accurate position estimates when evaluated on images from a previously unvisited area, (2) to reduce the search time, our method can first be used at a coarser scale to generate a slightly less accurate position estimate, and then the position estimate can be refined at a finer scale, (3) the position accuracy of our method degrades gracefully when narrowing the camera field of view.

The author planned and participated in the conduction of the field trials, contributed to the idea and design of the method, implemented the algorithms for the baseline method and for generation of the training data, performed the training and the evaluation, and wrote the main part of the manuscript. 


\subsection{Other publications}

Parts of the material presented in this thesis also appeared in the author's licentiate thesis:

B. Grelsson. Global Pose Estimation from Aerial Images: Registration with Elevation Models. Licentiate thesis No. 1672.

Linköping University Electronic Press, 2014. ISBN: 978-91-7519279-6.

The following other publications by the author are related to the included papers.

B. Grelsson, M. Felsberg, and F. Isaksson. "Global Pose Estimation of Aerial Images". In: SSBA (2013)

(Revised version of Paper A) 



\section{TAXONOMY OF VISION-BASED POSE ESTIMATION APPROACHES}

This chapter gives an overview of the main concepts of vision-based pose estimation methods and classify the methods based on the type of reference data used for registration. The classification is taken from Brejcha and Cadík [7], and it is illustrated in figure 2.1 with a flowchart taken from Paper F. In order to explain the classification of the methods, I will use examples of how we humans visually localize ourselves in our everyday life to aid our navigation and relate these concepts to the methods in the field of computer vision. After explaining the classification flowchart, the proposed methods in this thesis are briefly discussed and positioned in the flowchart.

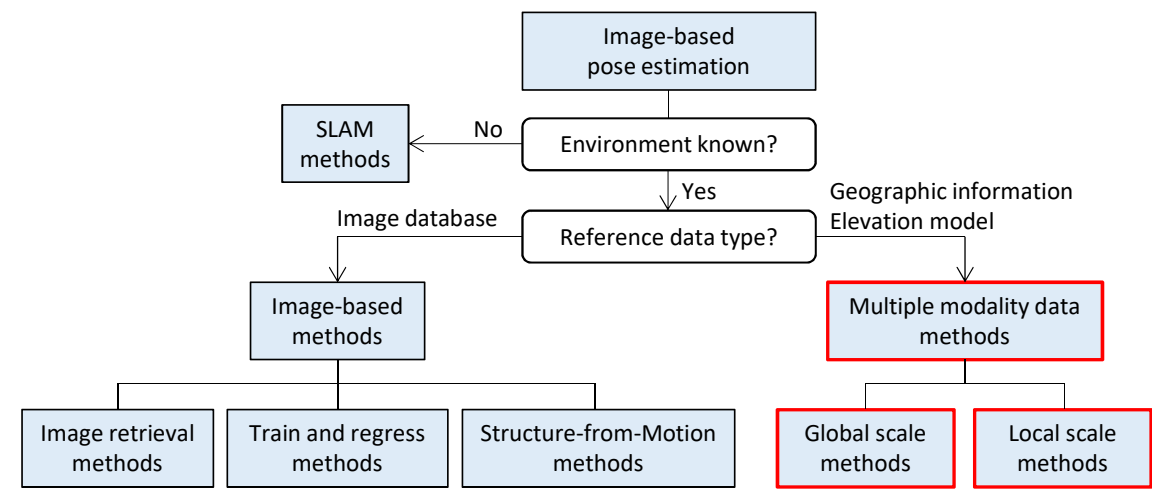

Figure 2.1: Flowchart of vision-based pose estimation methods from Paper F. The proposed methods in Papers A, C, E and F are local scale methods utilizing multiple modality data. The proposed method in Paper B is a global scale method using multiple modality data. 


\section{$2.1 \quad$ Image-based methods}

When we walk to school or ride our bicycle to work, we have probably already gone that very same route hundreds of times. Hardly reflecting upon it, we recognize objects like intersections, buildings, and traffic signs along the route to quickly confirm where we are, in what direction to proceed, and where to make turns. We check with our "visual memory bank" for previous images to do the localization. Over time, we have learned which visual cues along the route that are time invariant and useful for navigation and which ones that are temporary and only act as visual distractors and hence can be neglected. We have also learned how the visual memories are related to each other geographically in the global world, i.e. the visual memories are globally geo-tagged.

In computer vision, this group of methods for localization is called imagebased methods, i.e. image information is matched or registered with previously captured images from known locations in the same area. The image-based methods require a large database of geo-tagged images. In an urban environment, the image database can often be generated from public photographs or street-view images captured from cars. The database enables image retrieval methods for localization. The location of a query image is inferred by retrieving similar images from the database using various matching algorithms such as Bag-of-Words and hashing approaches [1], [48], [10]. Another option for localization is Train and regress methods where the image database is used to train a classifier and then directly regress the location of the query image [36], [58], [17]. An image database also enables 3D reconstruction of the scene using Structure-from-Motion. Various techniques have been proposed to align the query image with the 3D model to infer the camera location [34], [47], [39].

\subsection{SLAM methods}

When we fly to a city for the first time, we might take the metro from the airport to the hotel we will be staying at. When we exit the metro station and enter the streets, we first look at the surroundings and we often feel completely lost. We do not recognize the buildings around us and we do not know in what direction to go. But if we decide to start walking in one direction, we also start to store visual memories along that path. If we, after a while, turn around and walk back towards the metro station we will recognize the buildings we recently passed. We can localize ourselves in this local neighborhood (with the metro station as the origin), which we now have mapped with our visual memories. In computer vision, this way of localization is called visual SLAM (Simultaneous Localization And Mapping). There are numerous methods how to create and store the visual map and how to do the localization in this map. A recent overview of visual SLAM based methods can be found in [60]. 


\subsection{Multiple modality data methods}

Using visual SLAM, we can position ourselves in a local neighborhood. But to know how the metro station geographically is located relative to the hotel we are looking for, we need more information. To do a global localization, i.e. to localize ourselves in a world coordinate frame, we need to add information from another domain than just images. What we often do is to consult a map with street names, i.e. with artificial landmarks, and the location of the hotel in the same coordinate system. We may look for the position of the sun to decide if walking down the road means going north, south, east or west. What we have done is to use multiple modality data for vision-based localization. We have matched visual information with data from another domain for global localization.

The examples given above are all taken from an urban environment. The localization task becomes considerably harder in a natural environment, i.e. beyond urban areas, without any infrastructure or road network to guide your movements. Large image databases are rarely available in natural environments. This necessitates cross-domain matching of the query image with multiple modality data for localization. The old seafarers looked at the stars to orient and coarsely localize their ships. They used a very sparse set of "landmarks" for registration. In mountainous terrain, you may recognize the characteristic shape of two or more peaks, project the direction to them on a map and use triangulation or cross bearing for coarse localization. This procedure requires geographic information, in this case a terrain model or a digital elevation model (DEM) as reference data. DEMs are often used for cross-domain matching with images since they are readily generated worldwide from radar or image data captured from satellites.

The class of multiple modality data methods can be further divided into global scale methods, with the goal to estimate a coarse position within a large search area, and local scale methods aiming at accurate position estimates within a smaller search region. One example of a global scale method is [2] where the horizon line in the image is segmented and contour word descriptors are extracted. The authors infer the camera location within a $1 \mathrm{~km}$ radius by matching the contour words with a database generated from a DEM over the whole country of Switzerland.

The proposed methods in Papers A, E and F are all local scale methods utilizing multiple modality data to provide accurate position estimates. All three methods require an initial, approximate position to be known to limit the search area and processing time. In Paper A, a local height map is created using structure-from-motion. The height map is registered with a digital elevation model to infer the position and attitude. In Papers E and F, the horizon line is extracted in the omnidirectional image. The horizon line is registered with the geometric horizon line from a DEM to provide accurate position estimates. 
The methods proposed in Papers B and $\mathrm{C}$ also utilize multiple modality data to provide attitude estimates. In Paper B, the horizon line in the image is implicitly matched with a simplistic spherical earth model to provide an approximate global camera attitude. It is a global scale method since it can be applied anywhere on earth. In Paper C, the attitude estimate is refined by registration of the horizon line in the image with the geometric horizon line from a DEM. It is a local scale method since it requires an initial approximate position to be known.

\section{Pose estimation methods - system components}

In order to design, develop and evaluate vision-based pose estimation methods, a number of system components or building blocks are required. The system components utilized in the pose estimation methods in this thesis are presented in the sequel. The first component, which is central in all visionbased pose estimation methods, describes how to geometrically interpret the information in the camera image. This geometric interpretation is done using camera models. 


\section{Camera Models}

Vision-based pose estimation relies on the fact that accurate mathematical relationships can be established between $3 \mathrm{D}$ points in a world coordinate frame and their corresponding image coordinates. As the name suggests, camera models are used to provide a mathematical model how light rays from an object are propagated through the camera lens to the sensor chip, where the rays create an image. This chapter describes the camera models employed in the papers included in this thesis.

In Paper A, cameras with a normal lens with fixed focal length was used. Normal in this context refers to the fact that the image is close to what we humans normally see with our own eyes. For this type of lens, a simple pinhole camera model with minor lens distortion corrections is often adequate. In Papers E and F, images from a group of cameras with normal lenses were stitched together to create a panoramic image. For a fisheye lens, which was used in Papers B and C, the true lens design is far more complex and this is also reflected in the fisheye lens model which mathematically is more involved than for a normal lens.

\subsection{Pinhole camera model}

The very first camera used to acquire an image, a camera obscura [56], used the principle of a pinhole camera. Light rays from the object passed through an infinitesimal hole and created an image on the wall inside a dark box. The geometry is illustrated in figure 3.1 where, for mathematical convenience, the image plane has been placed in front of the pinhole and not behind it inside the box. 


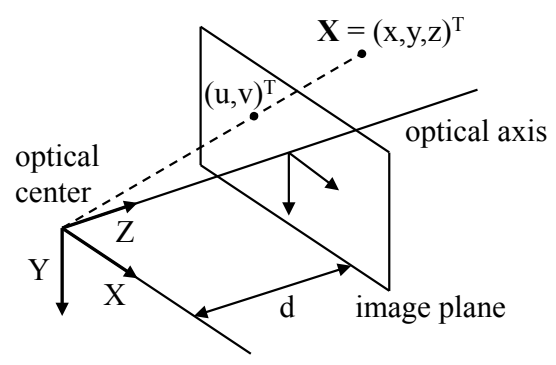

Figure 3.1: Pinhole camera model.

Consider a world 3D point $\mathbf{X}=\left[\begin{array}{lll}x & y & z\end{array}\right]^{T}$. If the distance from the pinhole to the image plane is denoted $d$, the image coordinates $\mathbf{u}$ of the point will be

$$
\mathbf{u}=\left(\begin{array}{l}
u \\
v
\end{array}\right)=\frac{d}{z}\left(\begin{array}{l}
x \\
y
\end{array}\right)
$$

It is often convenient to consider an image plane at unit distance from the pinhole, the so called normalized image plane. The normalized image coordinates are given by

$$
\mathbf{u}_{\mathbf{n}}=\left(\begin{array}{c}
u_{\mathrm{n}} \\
v_{\mathrm{n}} \\
1
\end{array}\right)=\left(\begin{array}{c}
x / z \\
y / z \\
z / z
\end{array}\right)
$$

In the real world, the pinhole is replaced with a thin lens (or rather a system of lenses) to allow for a larger aperture, letting more light through, and focus the light at a focal plane. Replacing the distance $d$ with the focal length $f$ of the lens, the pinhole camera model reads

$$
\lambda\left(\begin{array}{l}
u \\
v \\
1
\end{array}\right)=\left(\begin{array}{ccc}
f & \gamma & u_{0} \\
0 & \alpha f & v_{0} \\
0 & 0 & 1
\end{array}\right)\left(\begin{array}{l}
x \\
y \\
z
\end{array}\right)=\mathbf{K X}
$$

Since the sensor elements may not be exactly quadratic, an aspect ratio $\alpha$ has been introduced. The sensor may not be perfectly aligned with the lens allowing for a skew angle $\gamma$. For well manufactured lenses, $\alpha$ is very close to 1 and $\gamma$ is negligible. The origin in the image plane is not along the optical axis but has an offset $\left(u_{0}, v_{0}\right) . \lambda$ is a scaling parameter. The linear mapping $\mathbf{K}$ is called the intrinsic camera matrix.

In general, the camera coordinate system is not aligned with the world coordinate system. Their interrelationship is described by a rotation matrix $\mathbf{R}$ and a translation vector $\mathbf{t}$. These two parameters are called extrinsic camera parameters. If we define the camera matrix

$$
\mathbf{C}=\mathbf{K}[\mathbf{R} \mid-\mathbf{R t}]
$$


we can formulate a linear mapping of a world point to its image coordinates as

$$
\lambda\left(\begin{array}{l}
u \\
v \\
1
\end{array}\right)=\mathbf{C}\left(\begin{array}{c}
\mathbf{X} \\
1
\end{array}\right)
$$

\subsection{Lens distortion}

The camera model in the previous section assumes a rectilinear projection of points, i.e. straight lines in the world will be projected as straight lines in the image plane. For a real-world lens this is not perfectly true although often a very good approximation. Even though multiple-lens systems are used by the lens manufacturers, there still remains some imperfections called optical aberrations. The most common ones are radial and tangential distortion, and chromatic and spherical aberration.

The physical characteristics of the lens system introduce a phenomenon called radial distortion. Typically a square object will be imaged either with a barrel distortion or a pincushion distortion as illustrated in figure 3.2. The image will be more distorted further away from the center of the image. Tangential distortion in the image is created when the optical axis of the lens system is not perfectly aligned with the normal vector of the image sensor plane.

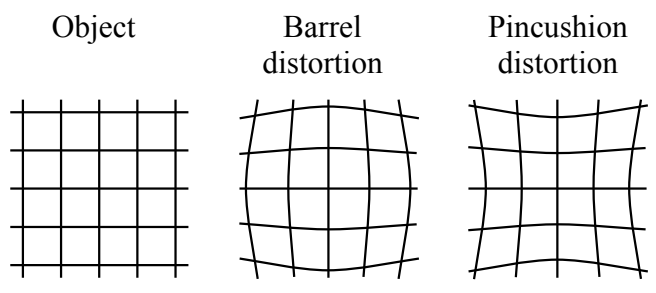

Figure 3.2: Radial distortion, barrel and pincushion.

The camera models used in Papers A, C, E and F took radial and tangential lens distortions into account. To mathematically compensate for radial and tangential lens distortions we first define a set of undistorted coordinates of each point in the normalized image plane,

$$
\begin{aligned}
& u_{\mathrm{u}}=u_{\mathrm{n}} \\
& v_{\mathrm{u}}=v_{\mathrm{n}}
\end{aligned}
$$

where the subscript $u$ means undistorted. We then define the radial distance $r$ as the distance from the origin in the normalized image plane. The total 
distortion in the $\mathrm{x}$ and $\mathrm{y}$ directions for each point in the normalized image plane is given by

$$
\begin{aligned}
& r^{2}=u_{\mathrm{u}}^{2}+v_{\mathrm{u}}^{2} \\
& d u=u_{\mathrm{u}} \sum_{i} k_{i} r^{i}+2 t_{1} u_{\mathrm{u}} v_{\mathrm{u}}+t_{2}\left(r^{2}+2 u_{\mathrm{u}}^{2}\right) \\
& d v=v_{\mathrm{u}} \sum_{i} k_{i} r^{i}+2 t_{2} u_{\mathrm{u}} v_{\mathrm{u}}+t_{1}\left(r^{2}+2 v_{\mathrm{u}}^{2}\right)
\end{aligned}
$$

where the first term (coefficients $k_{i}$ ) is the radial distortion and the latter terms (coefficients $t_{i}$ ) comprise the tangential distortion. We denote the set of lens distortion parameters with $D$. In Paper A, a polynomial of degree four was used for the radial distortion. For the fisheye lens in Paper C, a polynomial of degree eight (even terms only) was used.

The distorted coordinates in the normalized image plane are given by

$$
\begin{aligned}
& u_{\mathrm{d}}=u_{\mathrm{u}}+d u \\
& v_{\mathrm{d}}=v_{\mathrm{u}}+d v
\end{aligned}
$$

To obtain the final image coordinates of a pinhole camera with radial and tangential lens distortion, a mapping with the intrinsic camera matrix $\mathbf{K}$ is applied to the distorted coordinates.

Equations (3.7) and (3.8) give explicit expressions how to compute the forward lens distortion, i.e. going from undistorted to distorted coordinates. To compute the backward lens distortion, i.e. going from distorted to undistorted coordinates, iterative methods are normally used to solve a nonlinear equation system.

\subsection{Omnidirectional cameras}

A normal or perspective camera, as described in the previous section, is aimed at imaging straight objects in the world as straight lines in the image. There is a completely different class of cameras called omnidirectional cameras. As the name suggests, the aim is now to obtain an omnidirectional or $360^{\circ}$ view of the surroundings captured in one image.

The omnidirectional images used in this thesis were achieved in two physically very distinct ways. In Papers $\mathrm{E}$ and $\mathrm{F}$, the panoramic image in a cylindrical projection, figure $3.3(\mathrm{a})$, is created by stitching together five perspective images captured by five distinct camera sensors. In Papers B and C, the omnidirectional images are presented in a spherical projection, see figure 3.3(b). The image is created by a fisheye lens system and captured by a single sensor. The fisheye image is heavily distorted radially when projected on the image plane. 


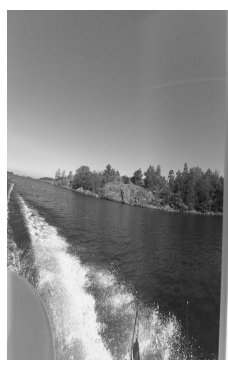

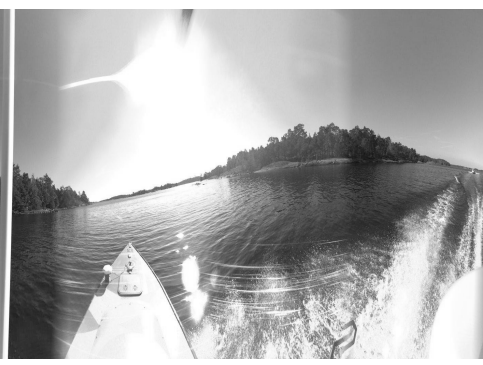

(a)

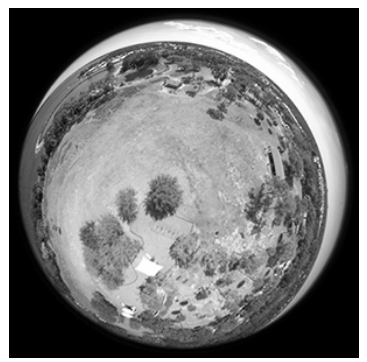

(b)

Figure 3.3: (a) Panoramic image in a cylindrical projection from Paper F. (b) Fisheye image from Paper B.

\section{Ladybug camera - cylindrical projection}

The Ladybug camera used in Papers E and F, figure 3.4, captures five perspective images equally spaced horizontally around its vertical axis. It actually captures six images, but the top camera was not used in our application imaging the horizon line around the USV.
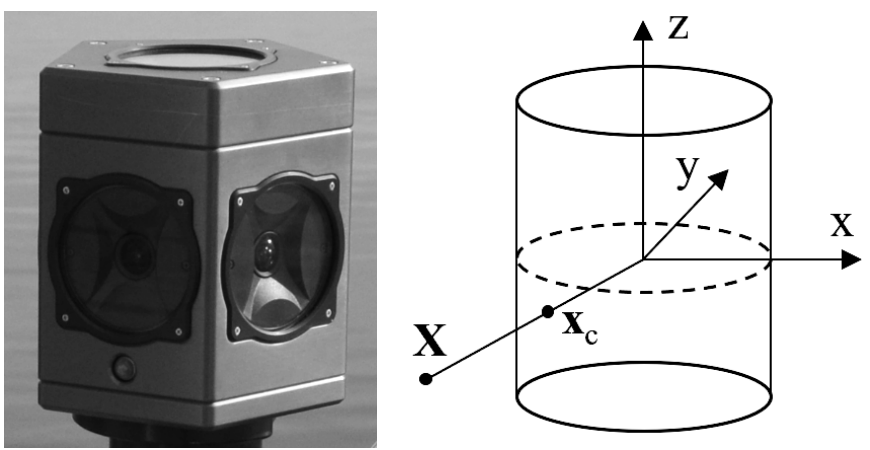

Figure 3.4: Ladybug camera (left), cylindrical projection (right).

The five individual images have a substantial horizontal overlap and the images have a quite large barrel distortion, see figure 3.5. The five images are rectified in accordance with their respective intrinsic camera calibration parameters. Based on the factory calibration of the extrinsic camera parameters, information from the five individual images are stitched together to generate a panoramic image in a cylindrical projection. In a cylindrical projection, a 3D world point $\mathbf{X}$ is projected onto the unit cylinder with radius 1 as point $\mathbf{x}_{\mathrm{c}}$, see figure 3.4. For the panoramic image exported from the Ladybug camera, the maximum elevation angle from the horizontal plane was set to $\pm 45^{\circ}$. 


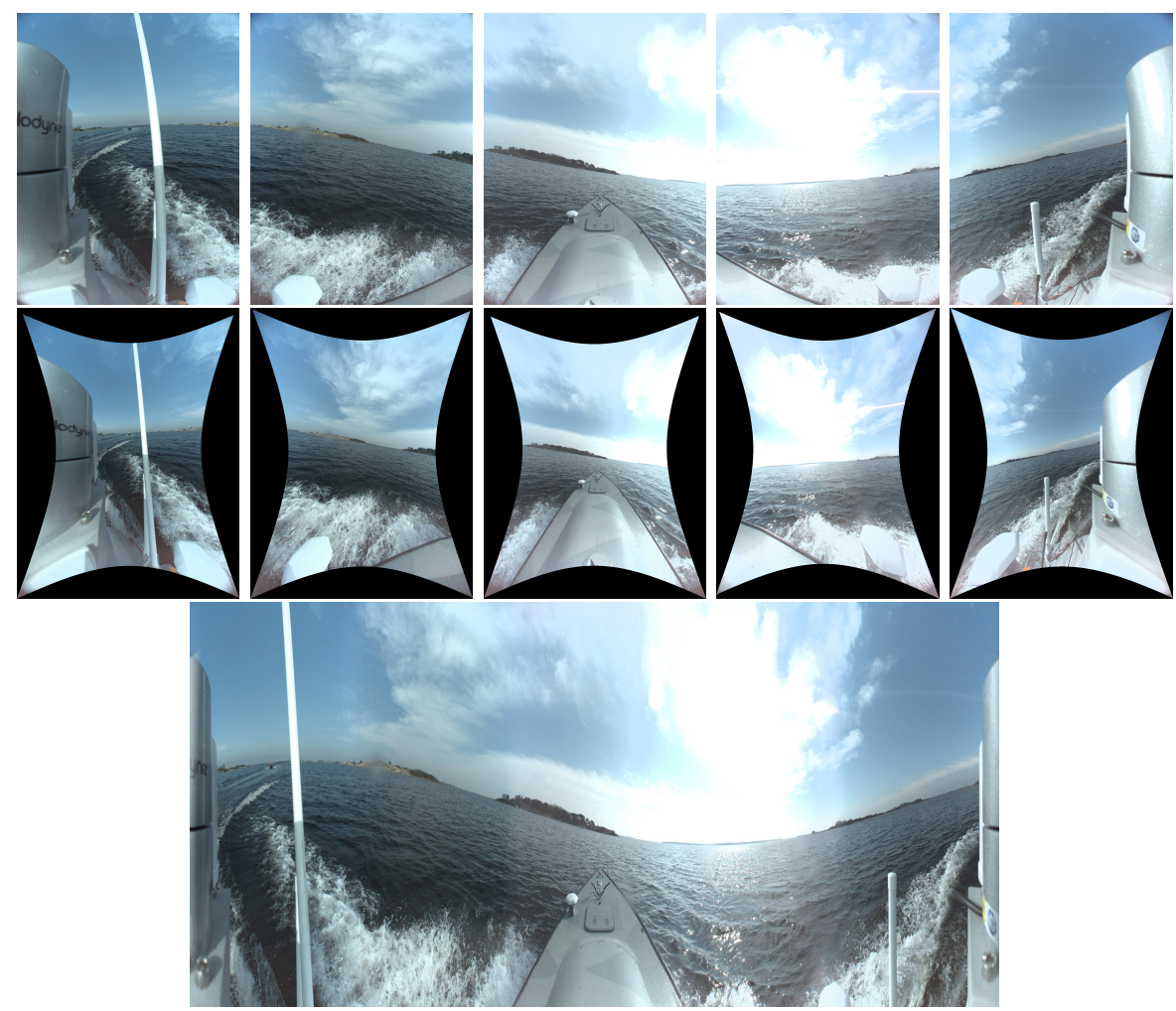

Figure 3.5: Ladybug camera raw images (top row), rectified images (middle row), and panoramic image in a cylindrical projection (bottom row).

\section{Fisheye camera - spherical projection}

A fisheye camera uses a system of lenses to achieve the aim of refracting light rays from roughly a hemisphere to a plane. A fisheye lens often suffers from noticeable chromatic aberration. A fisheye lens with a field of view larger than $180^{\circ}$ creates very typical images with a fisheye circle, a border line on the image plane outside of which no light rays will reach the sensor due to geometrical constraints, see figure 3.3(b).

The fisheye camera model used in Papers B and C is taken from [41]. It is based on the aim of the fisheye lens design - to image a hemisphere of world points onto a plane. First, a $3 \mathrm{D}$ world point $\mathbf{X}$ is projected onto the unit sphere, placed at the camera location, as point $\mathbf{x}_{\mathrm{s}}$, see figure 3.6. The point on the unit sphere is then projected onto the normalized image plane by a pinhole camera model with its optical center the distance $L$ from the center of the unit sphere and focal distance 1 (one) to the image plane. Next, radial and tangential lens distortions are applied. The final projection is a generalized camera projection $\mathbf{K}$ given by the intrinsic camera parameters. 


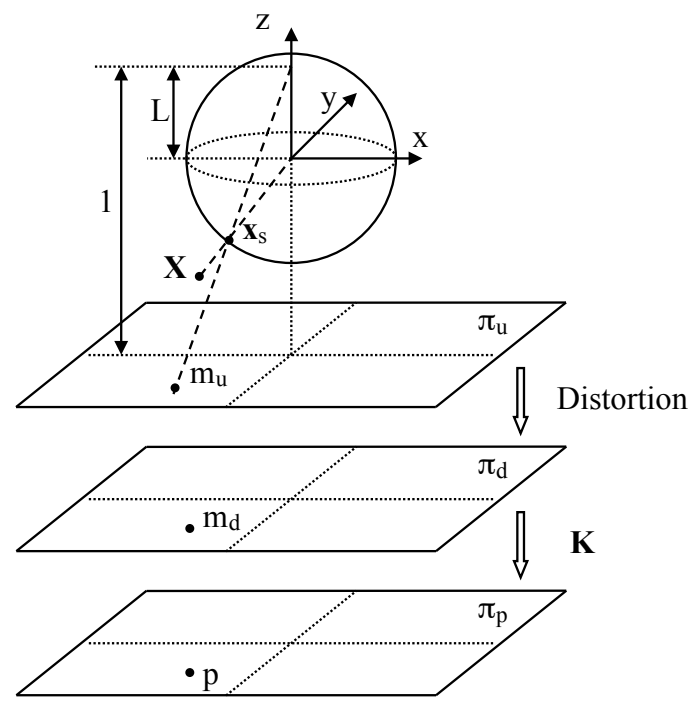

Figure 3.6: Fisheye camera model. Undistorted, normalized image plane $\pi_{u}$. Distorted image plane $\pi_{d}$. Image plane $\pi_{p}$.

\subsection{Camera calibration}

\section{Pinhole camera calibration}

The accuracy of the pose estimation methods we are interested in will of course rely on the accuracy of the camera calibration, i.e. how accurately we can determine the set of camera parameters. For calibration of a perspective lens with a pinhole camera model, the method by Zhang [62] is often used. The calibration object is a planar grid or checkerboard pattern with known dimensions. Images of the calibration object are captured in different orientations. From the linear mapping (homography) between the grid points in the object plane and the image plane, constraints can be established on the camera intrinsic parameters. If at least three independent orientations are used, all intrinsic and extrinsic camera parameters of a pinhole camera can be solved in closed form.

This calibration can be refined, also taking the lens distortion parameters into account, by minimizing the total reprojection error for all corner points,

$$
\left(\mathbf{K}_{\text {est }}, D_{\text {est }}\right)=\arg \min _{K, D} \sum_{i=1}^{n} \sum_{j=1}^{m}\left\|\mathbf{u}_{i j}-\tilde{\mathbf{u}}\left(\mathbf{K}, \mathbf{R}_{i}, \mathbf{t}_{i}, \mathbf{X}_{j}, D\right)\right\|^{2}
$$

The summation is made over the camera positions $i$ with the corresponding rotation $\mathbf{R}_{i}$, translation $\mathbf{t}_{i}$, the world corner points $\mathbf{X}_{j}$, the camera matrix $\mathbf{K}$ 
and the lens distortion parameters $D$. The symbol $\tilde{\mathbf{u}}$ denotes the projection of a world point onto the image plane and $\mathbf{u}_{i j}$ are the true image points.

The accuracy of the calibration will be dependent on the subpixel accuracy of the detector when extracting the corner points on the calibration pattern. The method is widespread since it is sufficiently accurate for most applications and because the checkerboard calibration pattern can be readily obtained from printers.

\section{Fisheye camera calibration}

Methods are also available for calibration of omnidirectional cameras with a checkerboard pattern [49], [41]. The method in [41] attempts to fit image data of a checkerboard pattern to the same fisheye camera model as presented in section 3.3. The method was used as a first stage for the camera calibration in Paper C. For the fisheye camera model, the mapping from the world checkerboard plane to the image plane is not linear and no closed-form solution can be obtained for the calibration parameters. In [41] they use reasonable assumptions on some calibration parameters as an initialization and then minimize a similar error function as in (3.9) using nonlinear optimization.

In Paper C, the calibration method in [41] was used to obtain an initial calibration subsequently refined using registration with true world 3D points. From the onboard navigation sensors an accurate ground truth for the vehicle 6 DoF pose was available. Given the world 3D position of the camera, a geometric horizon projected onto the unit sphere could be computed from DEM data. If horizon pixels can be extracted from the images, all information is available to compute a refined camera calibration using (3.9). The calibration method proposed in Paper $\mathrm{C}$ solved a dual formulation, i.e. it minimized the distances between the corresponding points on the unit sphere and not on the image plane.

\section{Method and component summary}

Utilizing camera models and camera calibration, we now have methods and tools to geometrically interpret the information in single camera images. A natural extension is to aggregate information over an image sequence. The next system component to be presented, where geometric information from two or more images is combined, is called multiple-view geometry. 


\section{4}

\section{MULTIPLE-VIEW GEOMETRY}

When a camera mounted on an airborne vehicle or a surface vessel captures images at high frame rates, there will generally be a substantial image content overlap between successive images. Geometrically, the combined image content from an image pair can be utilized analogously to how our human vision system uses stereo images. In the same way that we humans can determine distances and directions to objects within our view, the same information can be determined from two images if we know the stereo baseline (distance between the eyes) and how the cameras (eyes) are oriented. The principle of this two-view geometry, or epipolar geometry, is one of the keystones in computer vision and the fundament for vision-based reconstruction of 3D structures.

This chapter describes the basic concepts of epipolar geometry and the principle behind the dense 3D reconstructions used to generate the digital elevation models employed as reference data in this thesis. An example of two successive aerial images from a flight trial in Paper A, with some image point correspondences is shown in figure 4.1.

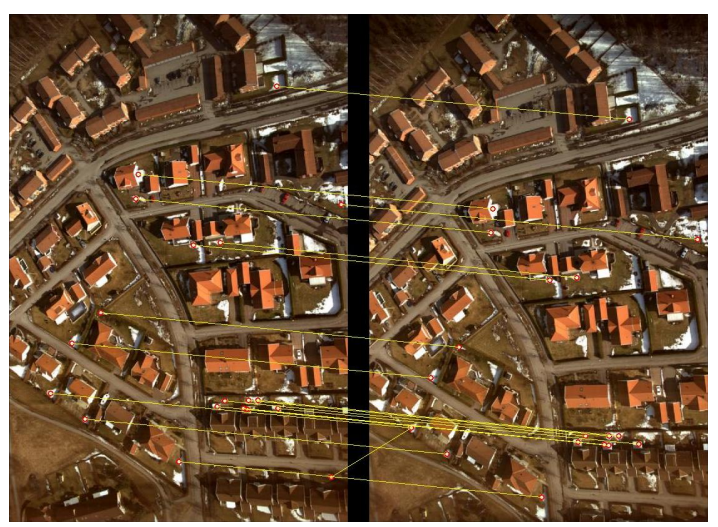

Figure 4.1: Image point correspondences and one feature matching outlier in two successive images from a flight trial. Image from Paper A. 


\subsection{Epipolar geometry}

The classical way to explain the concept of epipolar geometry is to consider the geometry in figure 4.2. Two pinhole cameras, located at positions $\mathbf{O}_{1}$ and $\mathbf{O}_{2}$, are imaging the same world point $\mathbf{X}$. The two cameras may be the same physical camera that has been moved or two different cameras, but the camera center locations need to be distinct. The projection of the world point $\mathbf{X}$ on the two image planes will be at $\mathbf{u}_{1}$ and $\mathbf{u}_{2}$ respectively. But $\mathbf{u}_{1}$ will also be the image point of all points on the $3 \mathrm{D}$ line passing through $\mathbf{O}_{1}$ and $\mathbf{X}$, e.g. the world points $\mathbf{X}^{\prime}$ and $\mathbf{X}^{\prime \prime}$. In the second camera, this $3 \mathrm{D}$ line will be imaged as the line $\mathbf{l}_{2}$ called an epipolar line. Repeating this process for other world points, it can be shown that all epipolar lines in the second image will intersect at a point $\mathbf{e}_{2}$ called the epipole. The epipole $\mathbf{e}_{2}$ is also the image point in the second image of the camera center $\mathbf{O}_{1}$.

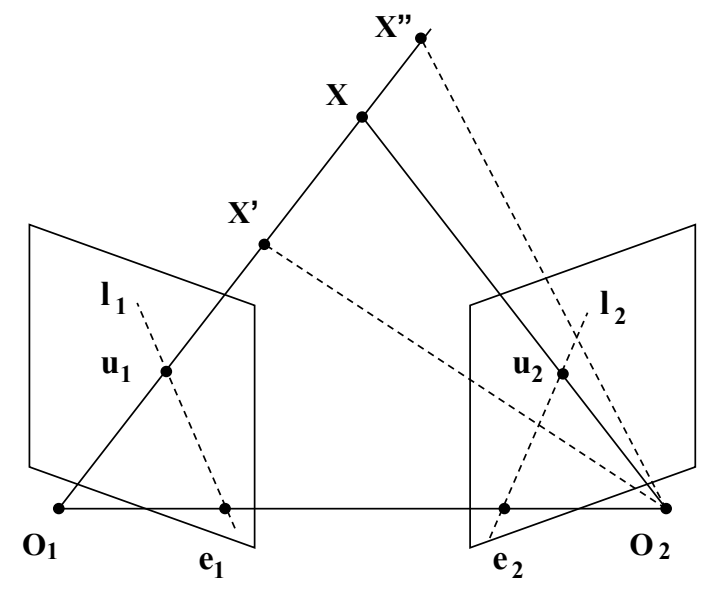

Figure 4.2: Epipolar geometry.

The constraint that an image point in the first image must lie on an epipolar line in the second image is known as the epipolar constraint and can mathematically be expressed as

$$
\mathbf{u}_{1}^{T} \mathbf{l}_{2}=\mathbf{u}_{1}^{T} \mathbf{F} \mathbf{u}_{2}=\mathbf{0}
$$

$\mathbf{F}$ is called the fundamental matrix and is a $3 \times 4$ matrix with seven degrees of freedom. A thorough mathematical derivation of the epipolar constraint and how the matrix $\mathbf{F}$ is related to the camera matrices $\mathbf{C}_{1}$ and $\mathbf{C}_{2}$ can be found in [31].

If two images have been captured in distinct locations with the same calibrated camera, the expressions for the epipolar constraint can be simplified further. We now denote the normalized coordinates of a point in the first 
image as $\mathbf{u}=\left[\begin{array}{lll}u & v & 1\end{array}\right]^{T}$ and in the second image as $\tilde{\mathbf{u}}=\left[\begin{array}{lll}\tilde{u} & \tilde{v} & 1\end{array}\right]^{T}$. For corresponding image points with a calibrated camera, the epipolar constraint can be expressed as

$$
\mathbf{u}^{T} \mathbf{E} \tilde{\mathbf{u}}=0
$$

The matrix $\mathbf{E}$ is called the essential matrix and can be decomposed as

$$
\mathbf{E}=\left[\mathbf{t}_{12}\right]_{\mathrm{x}} \mathbf{R}_{12}^{T}
$$

where $\mathbf{R}_{12}$ and $\mathbf{t}_{12}=\left[\begin{array}{lll}t_{x} & t_{y} & t_{z}\end{array}\right]^{T}$ are the relative rotation and the translation of the camera between images 1 and 2 , and $[\cdot]_{\mathrm{x}}$ denotes the cross-product operator meaning that

$$
\left[\mathbf{t}_{12}\right]_{\mathrm{x}}=\left(\begin{array}{ccc}
0 & -t_{z} & t_{y} \\
t_{z} & 0 & -t_{x} \\
-t_{y} & t_{x} & 0
\end{array}\right)
$$

The essential matrix has five degrees of freedom. This means that the matrix $\mathbf{E}$ can be computed from a set of five corresponding image points. Since $\mathbf{R}_{12}$ and $\mathbf{t}_{12}$ jointly have six degrees of freedom, it also means that from corresponding image points it is feasible to compute the relative rotation and the direction of the translation vector but not its magnitude. This is known as the scale ambiguity, i.e. the size of objects in the two images cannot be deduced from image information alone. An implementation of a five-pointsolver for $\mathbf{E}$ can be found in [43] which also presents how to decompose the essential matrix to a rotation and a translation.

\subsection{Local pose estimation}

The pose estimation method in Paper A is based on the computation of a local height map from two images and registration of the height map with elevation data from the area. To compute the local height map, the relative rotation and translation between the two images need to be determined.

From section 4.1 we know that the essential matrix $\mathbf{E}$ can be computed from at least five image correspondences. In the problem formulation in Paper A, an airborne monocular camera was supported by an inertial measurement unit (IMU) providing very accurate estimates of the relative rotation between images. If the rotation matrix $\mathbf{R}_{12}$ is known, the epipolar constraint can be expanded using $(4.3-4.4)$ to yield a linear equation system for the translation $\mathbf{t}_{12}=\left[\begin{array}{lll}t_{x} & t_{y} & t_{z}\end{array}\right]^{T}$ given by

$$
\left[\begin{array}{c}
\left(r_{12} u+r_{22} v+r_{32}\right)-\tilde{v}\left(r_{13} u+r_{23} v+r_{33}\right) \\
\tilde{u}\left(r_{13} u+r_{23} v+r_{33}\right)-\left(r_{11} u+r_{21} v+r_{31}\right) \\
\tilde{v}\left(r_{11} u+r_{21} v+r_{31}\right)-\tilde{u}\left(r_{12} u+r_{22} v+r_{32}\right)
\end{array}\right]^{T}\left[\begin{array}{c}
t_{x} \\
t_{y} \\
t_{z}
\end{array}\right]=0
$$

where $r_{i j}$ are the components of the rotation matrix. This linear equation system can be solved in a least-squares sense using a singular value decomposition (SVD) from two or more image correspondences. 


\subsection{Structure from Motion}

Structure from motion (SfM) was used as a tool in the proposed method for global pose estimation in Paper A. A brief description of the concept of SfM and $3 \mathrm{D}$ reconstruction is given below.

From epipolar geometry, we know that the relative rotation and translation between two images of a calibrated camera can be computed from image correspondences. Contrary, if we do know the rotation and the absolute translation of the camera between images, it is possible to compute the $3 \mathrm{D}$ coordinate of the world point corresponding to the image points. In figure 4.2, consider the line passing through the points $\mathbf{O}_{1}$ and $\mathbf{u}_{1}$ and a second line passing through the points $\mathbf{O}_{2}$ and $\mathbf{u}_{2}$. The intersection between these two lines will yield the $3 \mathrm{D}$ coordinate of the world point $\mathbf{X}$. This method to determine the position of the world point from two image points is called triangulation. In reality, these two lines will rarely intersect due to e.g. image noise, nonperfect feature tracking/matching and often the 3D point is taken as the point where the distance between the two lines is the shortest.

When using stereo vision for $3 \mathrm{D}$ reconstruction of a scene, the reconstruction can either be made sparse or dense. A sparse reconstruction means that a limited set of corresponding image points (feature points) are used to compute $3 \mathrm{D}$ points. For a dense reconstruction, all image points jointly seen in the two images are used to compute the $3 \mathrm{D}$ points.

\subsection{Dense 3D reconstruction}

In Paper A, a dense 3D reconstruction was computed using a phase-based stereo algorithm, originally based on [21] and then further developed at SAAB. The corresponding image points along the epipolar lines are determined from phase information and not using distinct feature points. The disparities obtained are converted to distances based on the estimated stereo baseline, the distance between the two cameras. The $3 \mathrm{D}$ reconstruction is then orthorectified based on the assumed global pose of camera 1 to generate a local height map. An example height map from an urban area created from two images is shown in figure 4.3. A 3D reconstruction employing the same stereo algorithm but using tens of images from various views is shown in the same figure. Note the obvious quality improvement of the $3 \mathrm{D}$ reconstruction with more distinct building outlines when aggregating image information from a large number of views. 

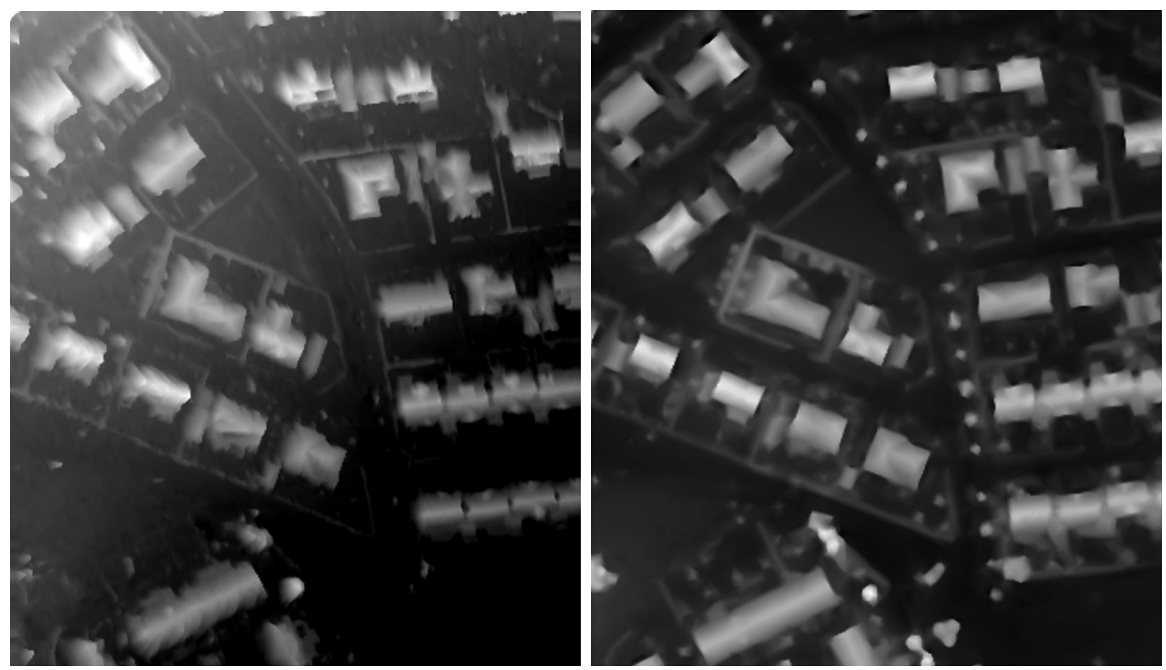

Figure 4.3: Dense 3D reconstruction of an urban area from two images (left) and from tens of images (right). The intensity represents the height above the ground. The images are taken from Paper A.

\section{Method and component summary}

Using camera models and multiple-view geometry, we can now geometrically interpret and combine image information from single and multiple camera images in a sequence. For vision-based pose estimation methods in natural environments, geometric information from images is often matched or registered with geographic information as reference data. The next system component to be introduced is geometric geographic information. 



\section{5}

\section{GEOMETRIC GEOGRAPHIC INFORMATION}

The vision-based methods for global pose estimation in this thesis employ a geo-referenced 3D model and terrain elevation data for registration. This chapter briefly describes the methods used to create these geometric geographic information models. It also describes how to compute the geometric horizon at close and far distances and discusses the considerations needed regarding the required resolution of the geographic models depending on the intended application.

\subsection{Digital Elevation Models}

Digital elevation models (DEMs) contain information on the altitude of the terrain as a function of ground position. The altitude data is often given over an equidistant XY grid with a certain resolution, but it may also be represented with an irregular triangular network. DEMs may e.g. be generated from several types of airborne sensors like lasers, radars and cameras.

When using a DEM it is of course essential to know the accuracy of the altitudes given. But equally important is to know whether the altitudes represent the true surface (including buildings and vegetation), the ground terrain or a mixture of these, see figure 5.1. This will depend on the sensor used as well as the postprocessing performed when generating the model. When matching altitudes computed from image data with a DEM, one error source may be the type of altitude the DEM actually represents, i.e. if it is a surface model (DSM), a terrain model (DTM) or something in between.

In Paper C, access to elevation data over a region as large as $\sim 400 \mathrm{~km} \times 400 \mathrm{~km}$ was required. This data could be provided by the publicly available database SRTM3 [42]. SRTM stands for Shuttle Radar Topography Mission and the data was captured from a space shuttle in the year 2000. 


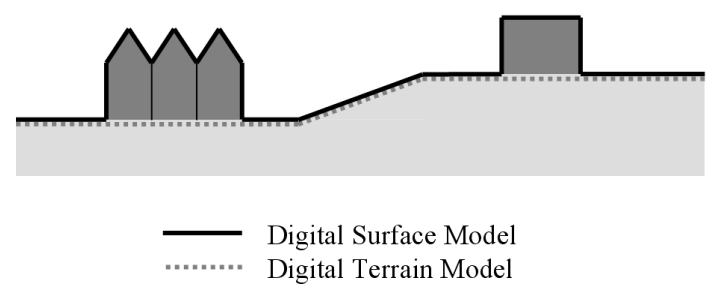

Figure 5.1: Digital Surface and Terrain Models.

The radar wavelength used was around $5 \mathrm{~cm}$ and the cell grid resolution is $\sim 90 \mathrm{~m} \times 90 \mathrm{~m}$. Although a large part of the microwaves at that wavelength was reflected on the surface of the objects within a cell, the altitudes given in the database will represent some sort of altitude average over the area covered within a cell. The absolute altitude accuracy is claimed to be about $8-10 \mathrm{~m}$.

\subsection{Vision-based 3D models}

Elevation models and 3D models may also be generated from aerial imagery using structure-from-motion. Images are captured over the desired area from an airborne vehicle which may be a satellite, an aircraft or a UAV, depending on the model resolution and coverage desired.

In Paper A, a 3D model from Vricon [55] was used. The georeferenced model was created from images captured from an aircraft scanning the city of Linköping at $600 \mathrm{~m}$ altitude. The $3 \mathrm{D}$ data is represented with a triangular mesh onto which texture from the images is overlaid to give a photorealistic impression. This feature also makes it possible to render images from a virtual camera at any position in the model, a method often used when trying to register a new image with the $3 \mathrm{D}$ model for global pose estimation. Figure 5.2 shows an example of a 3D model from a suburban area in Linköping. The $3 \mathrm{D}$ model is a digital surface model, since the aerial images capture the roof of the buildings and the top of trees. The resolution of the $3 \mathrm{D}$ model on the ground is about $0.1 \mathrm{~m}$.

In Papers E and F, similar digital elevation models from Vricon were used. These DEMs were generated from satellite imagery utlizing the same structure-from-motion process as above. A small part of the DEM used in Paper E is shown in figure 5.3(a). The resolution of the satellite image based models on the ground is $0.5 \mathrm{~m}$.

\subsection{Geometric horizon}

The proposed methods in Papers $\mathrm{C}, \mathrm{E}$ and $\mathrm{F}$ register the horizon line in the image with the geometric horizon computed from the DEM to infer the camera pose information. To compute the geometric horizon, we first make a 


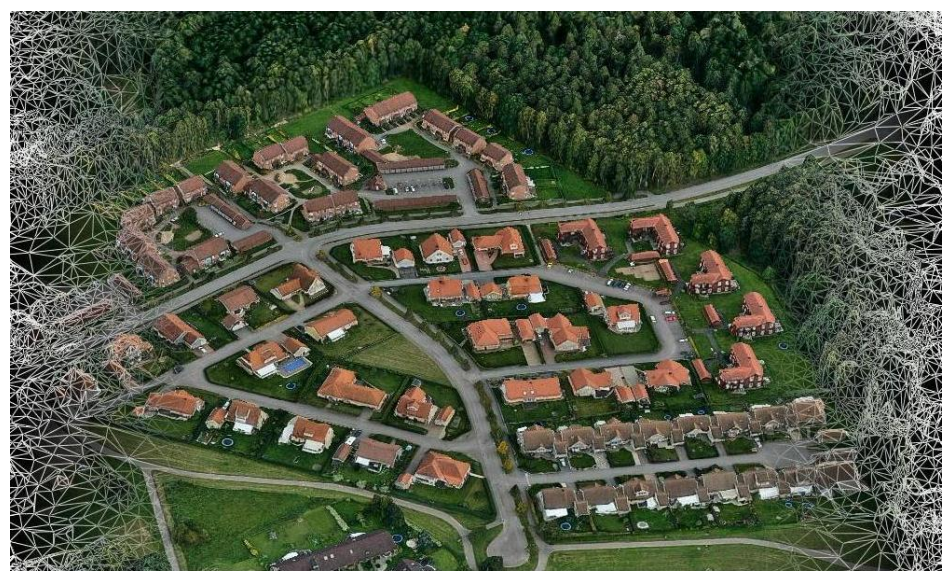

Figure 5.2: 3D model with triangular mesh and overlaid texture.

hypothesis of the global camera position and orientation. From this position we first consider a certain heading direction as in figure 5.3(a). Along this direction, we extract the altitude profile from the DEM, figure 5.3(b). We then compute the elevation angle to all objects along the ray direction figure 5.3(c). The maximum elevation angle is taken as the horizon point in this direction. This process is repeated in all directions around the camera. Projecting the horizon points onto the image plane will yield the geometric horizon, i.e. the expected horizon line from the DEM given the position hypothesis. In figure $5.3(\mathrm{~d})$, the geometric horizon is overlaid on an image that has been warped to compensate for the camera attitude angles.

In general, there is a very good fit between the geometric horizon line and the true horizon line in figure 5.3(d), but there are some small discrepancies. There are some obvious reasons for these deviations. The position and heading hypotheses may be slightly wrong. The DEM is not a perfect representation of the real world. There are errors in the $3 \mathrm{D}$ reconstruction process. The terrain changes over time, e.g. trees grow and change their appearance in different seasons, and there may be dynamic objects occluding the horizon.

\section{Ray refraction in the atmosphere}

In Papers E and F, the camera on the USV was placed about $1 \mathrm{~m}$ above the sea level. Assuming a spherical earth without topography, this means that the horizon will be located roughly $3 \mathrm{~km}$ away from the USV. For such a short distance to the horizon, a straight line approximation of the light ray from the horizon to the camera is adequate. However, for the airborne camera flying at altitudes between $300-600 \mathrm{~m}$ in Paper $\mathrm{C}$, the horizon will be located about $100 \mathrm{~km}$ away. For such a large distance, the effect of ray refraction has to be taken into account when using the horizon line for highly accurate attitude measurements. 


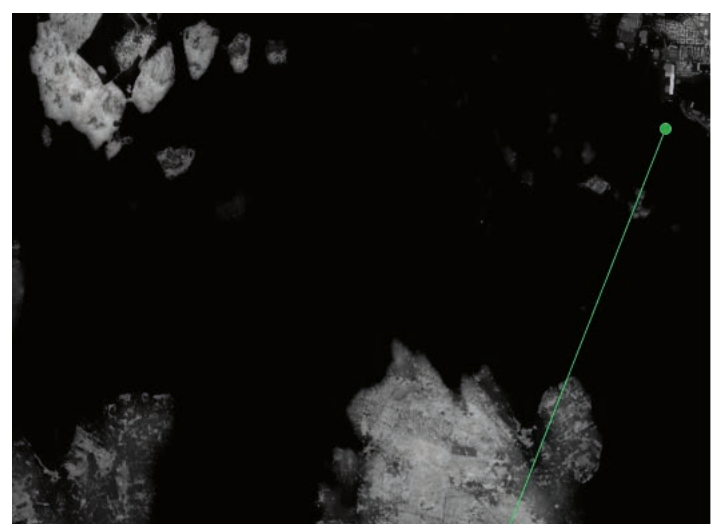

(a)

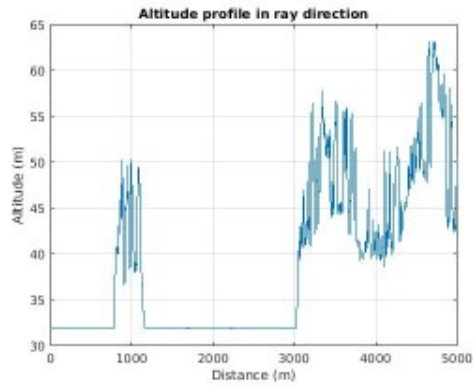

(b)

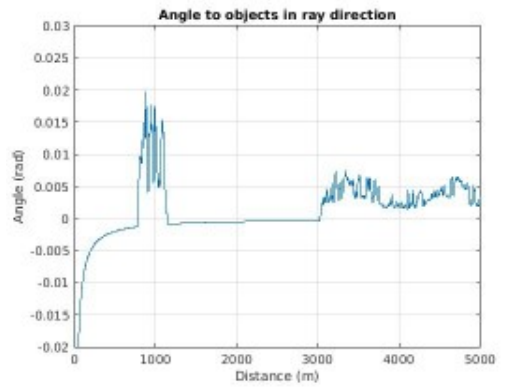

(c)

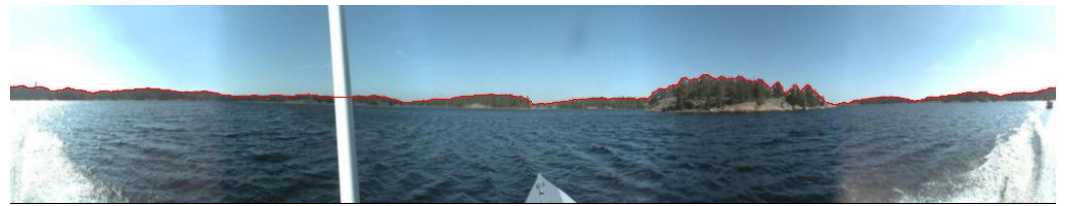

(d)

Figure 5.3: Computation of geometric horizon line. (a) Considered ray direction. (b) Extracted elevation profile. (c) Computed elevation angles. (d) Geometric horizon overlaid on image.

It is common knowledge that the air pressure drops with the altitude above the ground, i.e. that the air gets thinner at higher altitudes. We all experience this phenomenon when sitting in an aircraft that ascends or descends. It may be less known that this fact affects the exact location of the perceived horizon line on an image. It is not only the air pressure that varies with the altitude when the air gets thinner. The refractive index $n$ exhibits a similar behavior. The refractive index is higher close to the ground and drops with the altitude and equals unity in vacuum. This means that the light rays from an aircraft to the perceived horizon will not be straight lines but instead they will be slightly bent. This is illustrated in figure 5.4. 


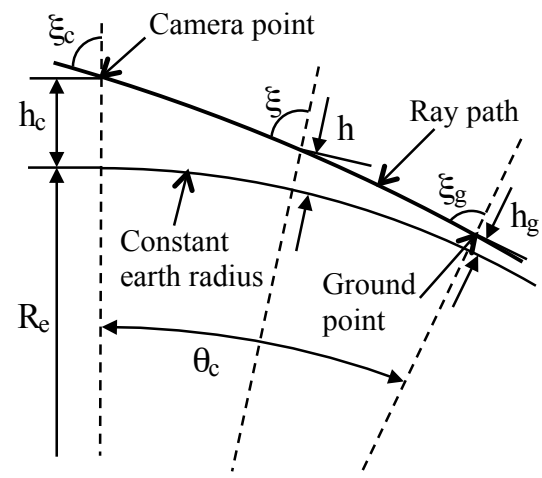

Figure 5.4: Refracted ray path in the atmosphere, from Paper C.

To quantitatively model the ray bending effect we first assume the earth to be a sphere with radius $R_{\mathrm{e}}$. Overlaid on this sphere, there is ground topography. We then use a spherically stratified model with thin radial layers of thickness $\Delta r$ and with constant refractive index. Using Snell's law, it can be shown [30] that a ray propagating through a spherically stratified model will follow a path which obeys the equation

$$
n r \sin \xi=n_{\mathrm{c}} r_{\mathrm{c}} \sin \xi_{\mathrm{c}}=n_{\mathrm{g}} r_{\mathrm{g}} \sin \xi_{\mathrm{g}}=k=\mathrm{constant}
$$

where $r=R_{\mathrm{e}}+h$ is the radius and $\xi$ is the incidence angle in the layer. The subscripts $c$ and $g$ denote the camera and the ground, respectively. The refractive index $n$ as a function of the altitude $h$ can be modelled, as a first order approximation, as

$$
n(h)=1+A \exp (-h / B)
$$

where $A$ and $B$ are not truly constants but vary slightly with the current air pressure and temperature, [30]. For the ideal horizon at sea level we have $h_{\mathrm{g}}$ $=0$ and $\xi_{\mathrm{g}}=\pi / 2$. Using (5.1) and (5.2), the incidence angle $\xi_{\mathrm{c}}$ of the ideal horizon on a camera at altitude $h_{\mathrm{c}}$ can be determined. In Paper C, equations are given for the angle $\theta_{c}$ from which the distance to the ideal horizon can be computed. Furthermore, Paper $\mathrm{C}$ also details how to compute the incidence angle to the perceived horizon when there are objects at altitudes above the ray path from the camera to the ideal horizon at sea level.

In Paper C, it is shown that using a straight line approximation when computing the geometric horizon, and thus neglecting the effect of ray refraction, a systematic error on the total tilt angle of about $0.05^{\circ}$ is generated. This is the same order of magnitude as the standard deviation of the attitude errors obtained with the method in Paper C. This shows that ray refraction must be taken into account for highly accurate pose estimation methods utilizing the horizon line at large distances. 


\section{DEM resolution}

When using elevation models as reference data for registration with image information, it is necessary to ensure that the resolution of the elevation model is sufficiently high such that it matches the image resolution. In Paper C, the SRTM3 elevation model with a grid resolution of $90 \times 90 \mathrm{~m}$ was used. In Papers E and F, a DEM generated from satellite imagery with a resolution of $0.5 \mathrm{~m}$ was used. Are these DEM resolutions adequate for their respective applications?

The airborne camera in Paper $\mathrm{C}$ was capturing images at an altitude of $300-600 \mathrm{~m}$ resulting in a horizon line at approximately $100 \mathrm{~km}$ distance. The radius of the fisheye circle on the image plane was about 2500 pixels. This means that 1 pixel on the image plane corresponds to a width of about $40 \mathrm{~m}$ of the horizon line at $100 \mathrm{~km}$ distance. This is about one half of the grid size of the SRTM3 model used, i.e. the DEM was too coarse to take full advantage of the image resolution. This is probably the reason why the attitude accuracy obtained with images downscaled a factor of two was almost as good as with the full image resolution. At that time, we did not have access to any DEM with higher resolution than the SRTM3 database with such a large area coverage.

The camera on the USV in Papers E and F was located about $1 \mathrm{~m}$ above the sea level resulting in a horizon line approximately $3 \mathrm{~km}$ away. This corresponds to viewing a circle with a periphery of roughly $20 \mathrm{~km}$. The panoramic images from the Ladybug camera were generated with 2048 pixels in the horizontal direction. This means that a pixel on the image plane corresponds to a width of about $10 \mathrm{~m}$ at a distance of $3 \mathrm{~km}$. The pixel resolution on the ground of the DEM used was $0.5 \mathrm{~m}$, i.e. the resolution was sufficient for the application. In reality, the horizon line was created by objects significantly closer to the USV than the ideal horizon. Calculations show that for objects at a distance of $150 \mathrm{~m}$, a pixel corresponds to a width of $0.5 \mathrm{~m}$, i.e. equal to the DEM resolution. For objects closer than $150 \mathrm{~m}$, the DEM used will not be sufficiently detailed to match the full image resolution.

\section{Method and component summary}

For the vision-based pose estimation methods, we now have methods to extract geometric geographic information from digital elevation models as reference data. In natural environments, the horizon is a frequently used landmark for registration with elevation data to infer the camera pose. The next system component to be presented is methods to detect and extract the horizon line in omnidirectional camera images. 


\section{HORIZON DETECTION}

The horizon line is often used by pilots as an attitude guide during take-off and landing. In the same manner, horizon detection is an intuitive means for us humans to determine the absolute attitude (pitch and roll angles). This insight has led to a large number of vision-based methods for localization and attitude estimation via horizon detection. This chapter describes how the horizon line can be detected and extracted in omnidirectional images using the concept of Hough voting.

The image shape of the horizon line will depend largely on the camera type used, see figure 6.1. For perspective cameras, the horizon (sky/ground border) will generate a contiguous rather straight line across the image. For fisheye cameras, the horizon will ideally form an ellipse on the image plane. For panoramic images in a cylindrical projection, the horizon will create an S-shaped curve on the image plane.

Irrespective of the camera type used, there are two main strategies for horizon detection in images. For one group of methods, sky/ground segmentation of the image is the key component. The segmentation may e.g. be based on pixel color and/or texture content. The segmentation may also be performed by training a convolutional neural network. Horizon detection methods based on sky/ground segmentation are found in [52] and [40] for perspective images and in [12] for omnidirectional images. CNN-based horizon segmentation can be found in [54], and was also used in Papers E and F. Once the horizon line has been determined from the segmentation process, its location in the image may be converted to attitude angles.

The second group of methods relies on the fact that the horizon often generates a distinct edge in the image. A commonly used method to detect edges is the Canny detector [8]. Horizon detection methods based on edge detection can be found in [4], [15] and [14] for perspective images. Papers B and $\mathrm{C}$ are the first papers proposing methods for horizon detection in fisheye images based on edge detection. One difficulty for edge based methods is to 


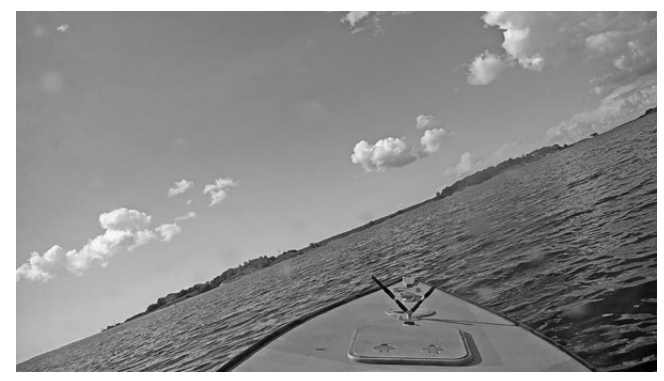

(a)

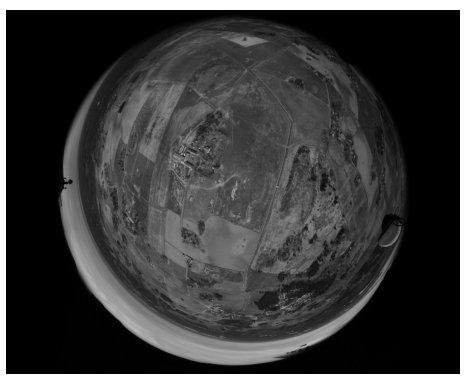

(b)
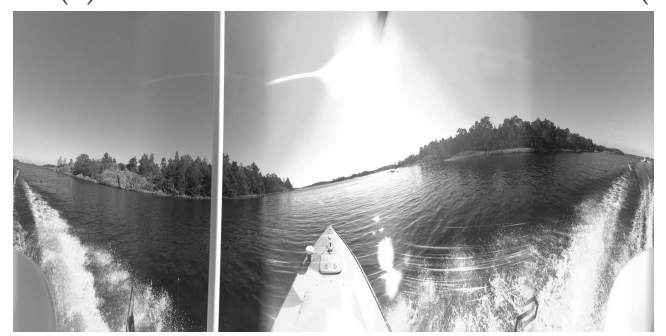

(c)

Figure 6.1: Horizon seen in (a) perspective image, (b) fisheye image in spherical projection, and (c) panoramic image in cylindrical projection.

select which edge pixels that originate from the horizon and which edge pixels that are generated by the remainder image scene content.

A common approach to detect lines or ellipses in images is to use the concept of Hough voting [33], a democratic process with a majority decision taken by all edge pixels. An advantage of Hough voting is that the sought shape can be detected although the complete shape is not present in the image. Hough voting was used in Papers B, C, E and F, and the main principle of the process, also known as the Hough transform, is given below.

The reason for choosing omnidirectional images for horizon detection and pose estimation is primarily that a substantially larger portion of the horizon line can be seen in the image compared to a perspective image. It is rather obvious that a more accurate pose estimate can be obtained when having information available on more than half of the full horizon line compared to seeing maybe only a tenth of it.

\subsection{Hough transform}

The Hough transform is a method for detecting a curve of a specified shape in an image. It exploits the duality between points on a curve and the parameters of that curve. The original work on the Hough transform was presented in [33] and was restricted to binary edge images to detect the shape of interest. 
Straight lines and circles are the most common shapes to be detected with the Hough transform. The generalized Hough transform [3] enables instances of any arbitrary shape in an image to be detected.

\section{Ellipse detection in a spherical projection}

In Papers $\mathrm{B}$ and $\mathrm{C}$, the objective was to detect the horizon in fisheye images to deduce the camera pitch and roll angles. For a fisheye camera and assuming a smooth earth, the horizon line will generate a circle when projected onto the unit sphere. On the image plane, it will form an ellipse, see figure 6.2.
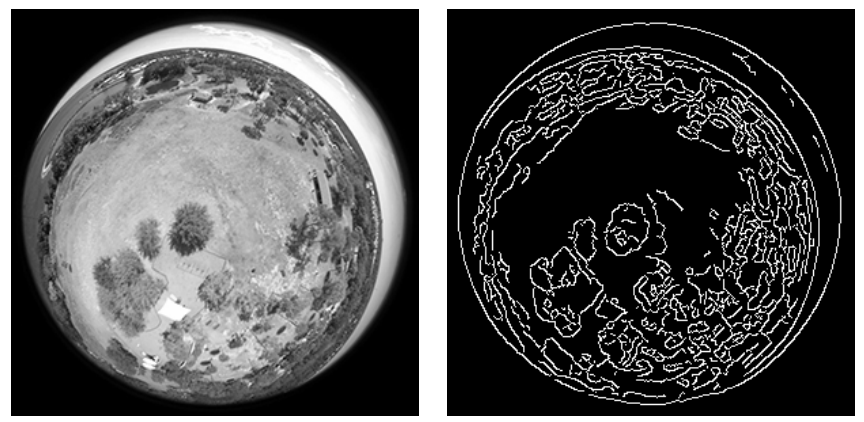

Figure 6.2: Fisheye image (left) and the corresponding edge map (right).

The available information to estimate the attitude angles is an edge map, a calibrated camera and we also assume the flight altitude $h$ to be known, e.g. from a pressure meter onboard the airborne vehicle. Somehow we want to exploit the location of all edge pixels and their gradient direction to vote for the camera attitude. Which is the most tractable parameter space for the Hough voting? We decided to perform the voting on the unit sphere and not on the image plane. Here are some qualitative justifications for that decision.

As a reminder, an ellipse has five degrees of freedom. One parameterization is the length of the major and minor semi-axes $a$ and $b$, the center point $\left(x_{0}, y_{0}\right)$ and a rotation angle $\gamma$ around the center point. The equation of an ellipse with these parameters is

$$
\left(\frac{x-x_{0}}{a} \cos \gamma+\frac{y-y_{0}}{b} \sin \gamma\right)^{2}+\left(\frac{y-y_{0}}{b} \cos \gamma-\frac{x-x_{0}}{a} \sin \gamma\right)^{2}=1
$$

The camera attitude can be expressed either as the pitch and roll angles, $\theta$ and $\phi$, or a tilt angle $\alpha$ around a rotation axis $\mathbf{n}_{\text {rot }}$. For a fisheye camera with its optical axis aligned with the gravity vector (vertical), the projection of the horizon onto the unit sphere will be a horizontal circle. The radius $r$ of the circle is determined by the camera altitude $h$ only. The radius of the horizon circle on the unit sphere will remain constant irrespective of the camera attitude. Being able to simply reduce the voting space one dimension 
when knowing the value of one input parameter, in this case the altitude $h$, is very advantageous.

For a vertical camera, the projection of the horizon onto the image plane is a circle, i.e. the length of the two semi-axes $a$ and $b$ are the same. When tilting the camera, the major axis of the ellipse will be parallel to the rotation axis $\mathbf{n}_{\text {rot }}$. The length $a$ of the major axis will increase with the tilt angle $\alpha$ whereas the length $b$ of the minor axis will decrease, i.e. the length of the two principal axes will vary both with the altitude $h$ and the tilt angle $\alpha$. This combined variation does not cater for an easy reduction of the voting space when knowing the altitude $h$.

Furthermore, we define the edge direction in a point $p$ on the image plane as $\left(-\nabla_{y}, \nabla_{x}\right)$, i.e. normal to the gradient direction. When projecting a point and its edge direction onto the unit sphere, the projected edge direction will constitute a tangent vector of the horizon circle plane in that point, see figure 6.3. $P$ is the projection of point $p$ onto the unit sphere, and $Q$ is a point on the unit sphere such that $P$ and $Q$ define a plane with radius $r(h)$.

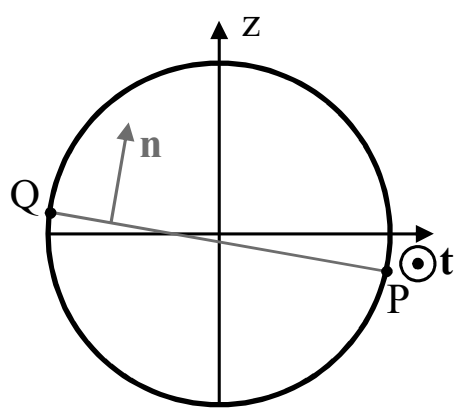

Figure 6.3: Estimate of the horizon normal unit vector $\mathbf{n}$ from edge points. The tangent vector $\mathbf{t}$ is directed out of the paper.

But if we have the projected point $P$ on the unit sphere, a tangent vector $\mathbf{t}$ of the horizon circle, and we also know the radius $r(h)$ of that circle, the normal vector $\mathbf{n}$ of the horizon circle plane can be readily computed using the cross product $\overrightarrow{P Q} \times \mathbf{t}$. The sought pitch and roll angles are then easily deduced from the normal vector direction. The explicit equations for these computations are given in Papers B and C. All edge pixels are given a vote and the location of the maximum score in the accumulator array is taken as the approximate horizon line.

On the image plane, the edge direction puts a constraint on the ellipse parameters which is obtained by differentiating (6.1). The equations and interpretation are far from being as trivial as for the tangent vector on the unit sphere. A second constraint is that the edge point shall lie on the ellipse and satisfy (6.1). Combined with the previous three constraints related to the length and direction of the ellipse axes, there is a total of five constraints 
which is sufficient to compute an estimate of the attitude angles. However, the mathematics for computing the attitude angles from the five constraints given by the ellipse shape on the image plane is truly intricate and not a tractable solution to the attitude estimation problem. This is the main reason for the decision to perform the Hough voting on the unit sphere and not on the image plane.

\section{Ellipse detection in a cylindrical projection}

In Papers E and F, the aim was to first detect the approximate horizon line in the panoramic image to infer the pitch and roll angles. The attitude angles were later used to warp the image to generate an image from a virtual level camera. The ideal horizon from a spherical earth with no topography will generate an S-shaped curve on the image plane in a cylindrical projection, see figure 6.4. The amplitude of the "S" and the location of the minimum will be dependent on the pitch and roll angles.
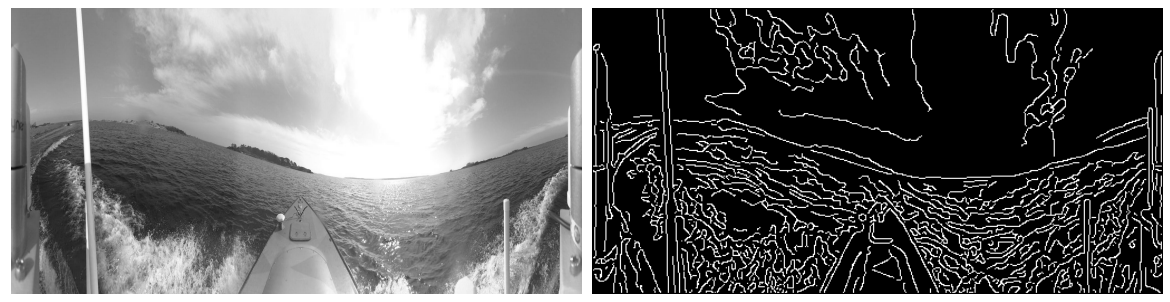

Figure 6.4: Panoramic image (left) and the corresponding edge map (right).

In principle, the horizon line could be searched for on the image plane using a generalized Hough transform. Again, we prefer to use the dual formulation and perform the Hough voting on the unit cylinder, since mathematically this is a more tractable solution. For a level camera, the ideal horizon will generate a horizontal circle when projected onto the camera fixed unit cylinder. When tilting the camera, the ideal horizon will generate a plane tilted with the camera pitch and roll angles when projected onto the camera fixed unit cylinder, see figure 6.5.

The normal vector of the tilted plane can now be estimated using Hough voting on the unit cylinder in a similar fashion as on the unit sphere described above. An edge pixel is projected onto the unit cylinder at a point $P$. The gradient direction of an edge pixel on the image plane is used to determine a tangent vector $\mathbf{t}$ on the unit cylinder. Their cross product is used to compute the normal vector $\mathbf{n}$ which is parameterized with the pitch and roll angles. Finally, all edge pixels are given a vote and the location of the maximum score in the accumulator array gives the estimated attitude angles. 


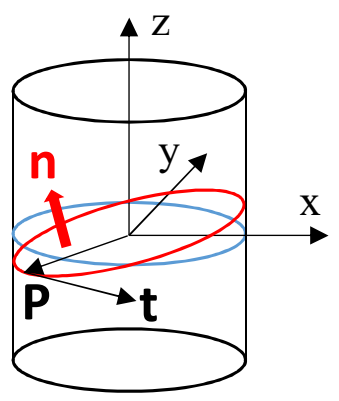

Figure 6.5: Projection of the ideal horizon onto a camera fixed unit cylinder for a level camera (blue) and for a tilted camera (red).

\subsection{Hough voting - considerations for real images}

For real world images, the horizon does not generate a perfect ellipse on the image plane and the circular shape on the unit sphere and the unit cylinder is also an approximation. It is mainly the true image scene content that causes the shape imperfections, but also image noise and camera model errors contribute to this deviation from the ideal shape. What considerations need to be taken to accommodate for these imperfections in the Hough voting?

In Papers B, C, E and F, where the Hough voting was carried out over a pitch and roll angle accumulator array, the shape and gradient direction imperfections imply that the computed attitudes for the different edge pixels will be spread out in a neighborhood around the true attitude value. The aggregated votes often create local maxima in the accumulator array and just picking the cell with the maximum score may lead to an erroneous estimate. Consequently, it is common to filter the accumulator array with a smoothing kernel prior to extracting the cell with the maximum value. This smoothing makes the attitude estimates more robust to the imperfections mentioned above.

\subsection{Extraction of horizon edge pixels}

\section{Aerial fisheye images}

In Paper B, the horizon detection in the aerial fisheye images and the attitude estimate were based on the assumption that the earth was a smooth sphere with no topography. Figures 6.6 (a) and (b) show an example image and the corresponding edge map used in the Hough voting. The estimated horizon is marked white in the edge map.

In Paper $\mathrm{C}$, the attitude estimate was refined by registration of the detected horizon line with the geometric horizon from a DEM. To perform the refinement, we need to extract only the edge pixels that originate from the 


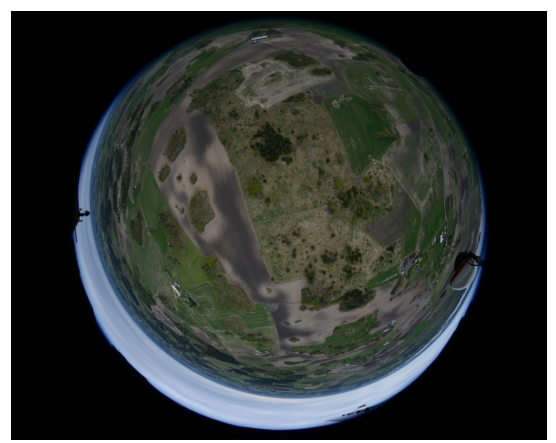

(a)

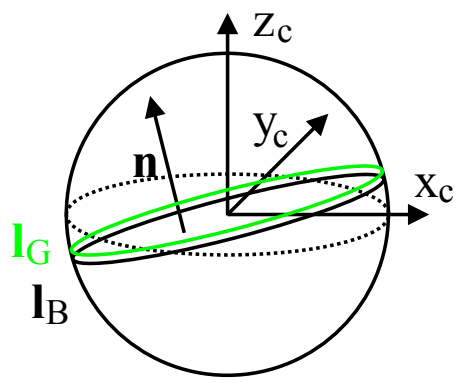

(c)

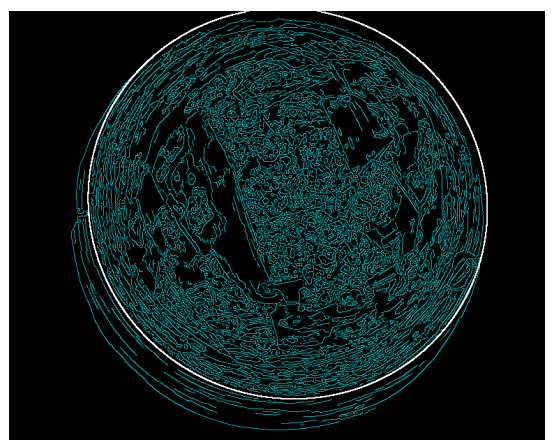

(b)

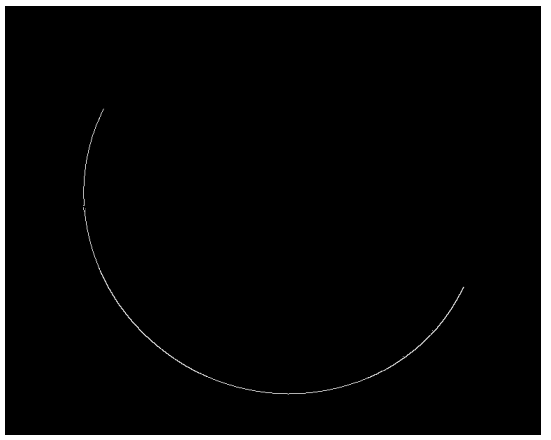

(d)

Figure 6.6: (a) Original fisheye image. (b) Canny edge map with estimated horizon from Hough voting in white. (c) Extract edge pixels in band between lines $l_{\mathrm{B}}$ and $l_{\mathrm{G}}$. (d) Extracted horizon edge pixels.

horizon in the image. This is done geometrically by reasoning as follows. For a perfectly calibrated camera and exact knowledge of the camera altitude, the ellipse on the image plane corresponding to the estimated horizon from the Hough voting will always be slightly smaller than the true perceived horizon on the image due to the topography on top of the ideal spherical earth. Thus, most of the true horizon edge pixels will be on or outside the estimated horizon on the image plane. Due to quantization effects in the edge detector (only integer pixels), some true horizon edge pixels may be $1 / 2$ pixel inside the estimated horizon. If the shift of the horizon due to topography is less than one pixel, it is sufficient to project the estimated horizon on the image plane and extract all edge pixels that are within a $3 \times 3$ matrix from the horizon pixels on the image plane.

For high resolution images, and when the ground elevation in the scene is large, the shift of the true horizon line due to the topography may be larger than one pixel. For the flight in Paper C, we could use DEM data (find highest altitude in the area) to compute an upper angular limit for the shift of the horizon to $0.4^{\circ}$ and denote it $\beta_{\text {lim }}$. This means that all true horizon pixels on 
the image plane will be projected onto the unit sphere in a thin band above the estimated horizon as given by the probabilistic Hough voting, figure 6.6 (c). The black line $\mathbf{l}_{\mathrm{B}}$ in the figure is the estimated horizon. The green line $\mathbf{l}_{\mathrm{G}}$ is generated by points that make an angle $\beta_{\text {lim }}$ with the horizon points. Explicit equations of the lines are given in Paper $\mathrm{C}$.

We project the band between the lines $\mathbf{l}_{\mathrm{B}}$ and $\mathbf{l}_{\mathrm{G}}$ onto the image plane to create a mask for potential horizon edge pixels. Since the edge detector gives integer pixel values, we include a 3x3 neighborhood around each projection point on the image plane in the mask. From the Canny edge image, we only extract the edge pixels within the mask for the subsequent attitude refining process. The extracted horizon edge pixels, figure $6.6(\mathrm{~d})$, are used in the subsequent registration with the geometric horizon for attitude estimate refinement.

\section{Panoramic images from USV}

In Papers E and F, the distance from the USV to the land objects creating the horizon line varied considerably, from less than $100 \mathrm{~m}$ to more than several kilometers. Depending on the scene, the horizon line could basically be located anywhere in the upper part of the image, see figure 6.7. Hence, there is no easy way to extract horizon edge pixels from a pure geometric argumentation. To extract the horizon edge pixels, we employ a registration method that is described in chapter 8 .

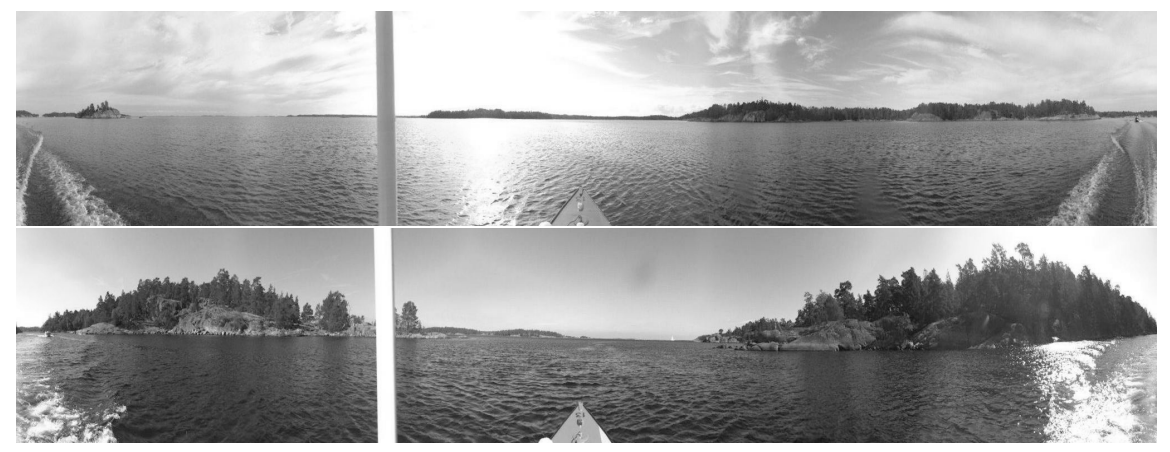

Figure 6.7: Panoramic images with the horizon line close to the center of the image (top) and close to the upper image border (bottom).

\section{Method and component summary}

In this chapter, methods have been described to detect and extract the horizon line in omnidirectional images using classical computer vision methods: Canny edge detection, Hough voting and camera models. The next system component to be presented is convolutional neural networks and how they can be used to extract information on the horizon line in the image. 


\section{Convolutional Neural NETWORKS}

As was pointed out in the introduction, deep learning and convolutional neural networks (CNNs) have revolutionized the field of computer vision over the last years. CNNs have significantly boosted the level of performance for a large variety of computer vision tasks such as image classification [37], [32], object detection [45], and object segmentation [9]. New network architectures, layer types, and methods to improve the training efficiency and the performance of the CNNs are proposed at a very rapid pace. Although existing network architectures vary greatly, there are some elements and operations that are used in most networks. A comprehensive summary of common methods and operations in deep learning networks can be found in [20].

This chapter first introduces the most common layer types used in CNNs. It then describes one of the layer types that is included in almost all CNNs, the nonlinear activation function, and the background to how it could be locally optimized in Paper D. Finally, the concept of transfer learning is presented and how it was employed in Papers $\mathrm{E}$ and $\mathrm{F}$ to estimate the camera orientation and to segment the horizon line in the image.

\subsection{Common layer types}

In this section, the most common layer types that are employed in CNNs will be briefly described. The terminology used is taken from [20].

Convolutional layers. In a convolutional layer, the input is convolved with a number of convolution kernels to generate the output called feature maps. The trainable kernel weights are normally shared within a layer and applied across the entire input. This parameter sharing is both memory and computationally efficient, and also leads to translational invariance, i.e. the 
convolutional layer will be activated in the same way to a certain feature in the image irrespective of its spatial location.

Activation function. The activation layer, sometimes called the detector stage, applies an elementwise activation to each of its inputs. In order to model complex image representations, it is essential to have nonlinear mappings in the network. Since convolutions are linear operations, the activation functions have the main responsibility for the nonlinear behavior of the network. Activation functions are addressed in more detail in section 7.2.

Pooling layers. The spatial pooling layer combines the inputs within a local neighborhood to produce a pooled output. The most common types are max pooling, where the maximum value within the local region is output, and average pooling, where the mean value over the region is output.

Fully connected layers. In a fully conncected layer, each neuron in the input is connected to all neurons in the output. These layers were the main component of early neural networks. The all-to-all neuron connectivity makes fully conncected layers computationally expensive for high-dimensional data.

Generally, a CNN can be divided into two main parts. The first part, often called the encoder, generates feature maps or feature descriptors to represent the input image at various levels of detail. The basic building elements of the encoder are convolutional layers, nonlinear activation functions and pooling layers. The second part of the CNN utilizes these image representations and is trained to output e.g. the image object class, the location of a tracked object or a pixelwise horizon segmentation. The second part in a classification network, the classifier, often comprises a few fully connected layers, activation layers and sometimes pooling layers. The final stage of a classification network is a softmax operation, which outputs the probabilities for each object class.

\subsection{Nonlinear activation functions}

In the early era of CNNs, nonlinear activation functions as the sigmoid and the tanh functions were used. However, they suffered from the vanishing gradient problem for large amplitude inputs which impeded the training capability of the networks. The more modern activation functions are derived from the Rectified Linear Unit (ReLU) [19]. The average response of ReLU is positive, and to reduce the bias shift variants such as the Leaky ReLU (LReLU) and Shifted ReLU (SReLU) were introduced. All these activation functions have a "knee", which led to the introduction of the continuously differentiable Exponential Linear Unit (ELU) [11]. The characteristics of ELU showed to increase the learning speed and to improve the classification performance of CNNs compared to previous activation functions. The definitions of the activation functions derived from ReLU are given in table 7.1. Parametric versions of these activation functions, where the hyperparameters are tuned during training, also exist. 
Table 7.1: Activation functions.

\begin{tabular}{|l|c|c|}
\hline Activation & $x>0$ & $x \leq 0$ \\
\hline ReLU & $\mathrm{x}$ & 0 \\
LReLU & $\mathrm{x}$ & $\alpha \mathrm{x}$ \\
SReLU & $\mathrm{x}$ & $\max (\mathrm{x},-1)$ \\
ELU & $\mathrm{x}$ & $\alpha(\exp (\mathrm{x})-1)$ \\
\hline
\end{tabular}

\section{Piecewise linear activation function}

In order to search for a superior activation function compared to ELU, we introduce a new formulation for a piecewise linear activation function in Paper D. The idea is to linearize the activation function in equidistant segments and to tune them during training to find the locally optimal shape of the activation function. Our formulation consists of two parts. First, a soft histogram of the input is formed as in [16], and, second, a weighted sum of the histogram is computed.

\section{Soft histogram}

The soft histogram is represented by an offset component, $o_{\nu}$, and a histogram component, $h_{\nu}$. An analytical formulation of the soft histogram is given as

$$
s(x)=\left[\begin{array}{c}
(x-\operatorname{floor}(x)-0.5) m_{\nu}(x) \\
m_{\nu}(x)
\end{array}\right]_{2 \times 2 \mathrm{~N}}=\left[\begin{array}{llll}
o_{0} & o_{1} & \ldots & o_{2 \mathrm{~N}-1} \\
h_{0} & h_{1} & \ldots & h_{2 \mathrm{~N}-1}
\end{array}\right],
$$

where $m_{\nu}(x)$ denotes the membership of the respective bins. The membership is 1 if the unit belongs to that bin and 0 for all other bins. The offset component is the signed distance from the bin center.

To illustrate the soft histogram concept, we use a total of $2 \times 4$ bins, i.e. 4 bins on each side of the origin, and consider the input range $[-4,4]$. To exemplify, the numerical input numbers -1.80 and 2.68 are used. The soft histogram output of these inputs will be

$$
\begin{gathered}
s(-1.80)=\left[\begin{array}{ccccccccc}
0 & 0 & -0.30 & 0 & 0 & 0 & 0 & 0 \\
0 & 0 & 1 & 0 & 0 & 0 & 0 & 0
\end{array}\right], \\
s(2.68)=\left[\begin{array}{llllllcl}
0 & 0 & 0 & 0 & 0 & 0 & 0.18 & 0 \\
0 & 0 & 0 & 0 & 0 & 0 & 1 & 0
\end{array}\right] .
\end{gathered}
$$

The output from the soft histogram is independent of the activation function, but any activation function can be represented or approximated by a weighted sum of the histogram output. 


\section{Weighted sum}

Different piecewise linear activation functions can be realized by varying the weights applied to the soft histogram outputs. For each activation function, we define a matrix $W$ with weights for the offset components and the histogram components as

$$
W=\left[\begin{array}{llll}
w_{o_{0}} & w_{o_{1}} & \ldots & w_{o_{2 N-1}} \\
w_{h_{0}} & w_{h_{1}} & \ldots & w_{h_{2 \mathrm{~N}-1}}
\end{array}\right] .
$$

The weights of the offset components correspond to the slope of the activation function, and the weights of the histogram components correspond to the bias level at the bin centers of the activation function for each linear segment. The weight matrix of the LReLU activation function with $\alpha=0.1$ thus will be

$$
W_{\text {LReLU }}=\left[\begin{array}{cccccccc}
0.1 & 0.1 & 0.1 & 0.1 & 1 & 1 & 1 & 1 \\
-0.35 & -0.25 & -0.15 & -0.05 & 0.5 & 1.5 & 2.5 & 3.5
\end{array}\right] .
$$

The output of the weighted sum is the sum of an elementwise multiplication of the soft histogram with the weight matrix

$$
y=\sum_{\nu} w_{o_{\nu}} o_{\nu}+w_{h_{\nu}} h_{\nu}
$$

The weighted sum outputs of the LReLU activation function for the two example inputs are illustrated in figure 7.1. The outputs are -0.18 and 2.68 as desired.

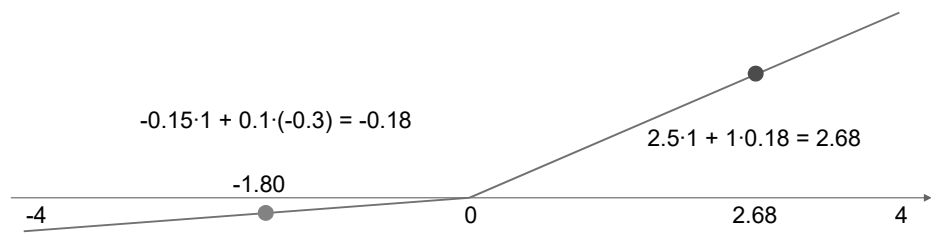

Figure 7.1: Weighted sum example with LReLU activation function.

Our formulation will generate a piecewise linear activation function for any values chosen as weights for the offset and histogram components. To obtain a continuous activation function it is necessary to put constraints on the weights. All offset weights (slopes) and one histogram weight (bias level) can be set independently, but the remaining histogram weights will be linearly dependent on the other weights. These constraints, which are to be enforced during backpropagation in the training process, are given in Paper D.

The piecewise linear activation function was applied during training of a classification network. The classification results were improved and the resulting shape of the activation function after tuning was noted to be ELUshaped, irrespective of its initialization, see figure 7.2. 


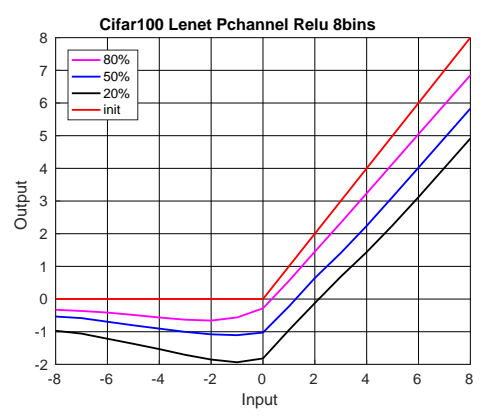

(a)

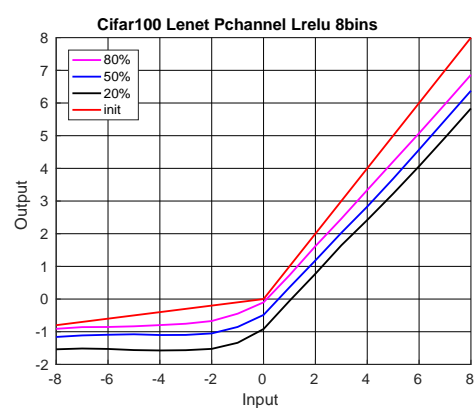

(b)

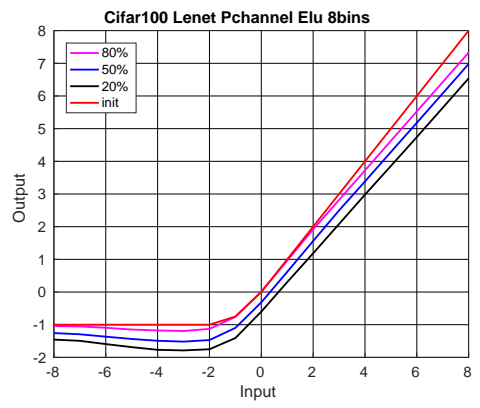

(c)

Figure 7.2: Results of tuned activation functions from Paper D. Initialization (red) and 20, 50 and 80 percentiles for tuned activation functions; ReLU (a), LReLU (b) and ELU (c).

Besides the ELU-shape, it was also observed that the tuned activation functions did not pass exactly through the origin, but the different neurons tend to return a variable negative output for zero input. This observation led to the introduction of a new activation function, the Shifted Exponential Linear Unit (ShELU). ShELU is an ELU-shaped activation function that has a random horizontal shift $\delta$. The random shift is individual for all neurons. Mathematically, ShELU is defined in table 7.2.

Table 7.2: ShELU activation function.

\begin{tabular}{|l|c|c|c|c|}
\hline Activation & Value & Region 1 & Value & Region 2 \\
\hline ShELU & $x+\delta$ & $x+\delta>0$ & $\alpha(\exp (x+\delta)-1)$ & $x+\delta \leq 0$ \\
\hline
\end{tabular}

The new ShELU activation function was used in experiments with classification networks on the dataset Cifar-100 in Paper D. Classification results were shown to be improved in comparison with ELU. The improvement was achieved when learning an individual bias shift $\delta$ for each neuron.

By introducing the activation function ShELU, with individual shifts for each neuron, we have implicitly generated individual biases for the convolu- 
tional layer feature map output. In other words, a convolutional layer with a shared bias level for each feature map output followed by a ShELU activation is equivalent to a convolutional layer with individual bias levels for each feature map output followed by an ELU activation. In modern deep learning frameworks, the normal procedure is to have one common bias level for each feature map output from a convolutional layer. Our results indicate that an individual bias term could improve the classification performance. A drawback is that individual biases come with an increase in number of weights and memory required.

\subsection{Transfer learning}

Transfer learning is a technique that is widely used within the field of deep learning. Transfer learning means that a network model is trained for one base task, and the trained model is then reused as the starting point to solve a second application task. There are two main drivers for using transfer learning. First, training on the application task can be made with a significantly smaller number of training images compared to training the full network from scratch. Provided that the base task is general enough, the learned model only needs to be fine-tuned when training on the application task. Second, there is a large gain in training time and thus saving computational resources when solving the application task. Training the network on the base task with a huge dataset may take weeks on dedicated hardware. This training time can be saved when using transfer learning for the application task.

The concept of transfer learning was employed in Papers E and F, where two CNNs were used to predict the camera orientation and to segment the horizon line in the images, respectively. In both applications, we use a ResNet50 network [32], that had been pretrained on the ImageNet dataset [13], as the encoder. For the two CNNs called HorizonFinder and HorizonSegmenter, the Resnet50 network provides feature descriptors and we then add some convolutional layers, pooling layers, activation functions and a fully connected layer to train and predict the orientation angle output, and the pixelwise horizon line, respectively, see figure 7.3

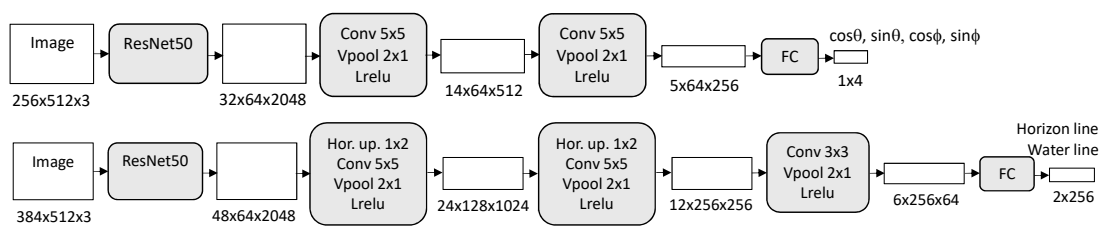

Figure 7.3: Network design for the CNNs HorizonFinder (top) and HorizonSegmenter (bottom) in Papers E and F. The numbers denote the input and output size $(\mathrm{H} \times \mathrm{W} \times \mathrm{C})$ for each network layer. 
Thanks to transfer learning, the two CNNs could be successfully trained and applied to their respective application tasks despite not having more than about 3000 labelled training images from the archipelago. This illustrates that a base network (ResNet50) pretrained on ImageNet with more than a million labelled training images can produce very generalized feature descriptors that can constitute the basis for a variety of application tasks.

\section{Method and component summary}

In this chapter, convolutional neural networks have been introduced. CNNbased methods to extract image information on the horizon line have been presented, in addition to the methods employing classical computer vision algorithms described in the previous chapter. For vision-based pose estimation in natural environments, the extracted geographic information from the images needs to be matched or registered with geometric information from the elevation models. This matching is accomplished with the next system component to be presented, registration methods. 



\section{8}

\section{REGISTRATION METHODS}

The output from the vision-based methods proposed in this thesis is a global pose estimate. Global in this context means that the estimated pose is given in a world coordinate frame, e.g. the WGS84 or UTM coordinate systems. To achieve the aim of global pose estimation, we must somehow find a relationship between the image content and the geometric geographic information provided in a world coordinate frame. This process is called registration, i.e. transforming different sets of data into one common coordinate system.

An overview of the main concepts for registration of images with different types of reference data was given in chapter 2 . This chapter describes the registration methods employed in this thesis in more detail.

\subsection{D-3D registration}

\section{Paper A - registration of height information}

In Paper A, the problem formulation assumed that we had a textured 3D model as reference data, see figure 5.2. The 3D model was generated from images captured in the Linköping area in the summer 2008. The query images, for which the camera pose was to be estimated, were captured in a flight trial in March 2010 when there was still some snow on the ground. This is a common situation, the geographic information (3D model) and the query images are from different instances in time and the registration method must be able to cope with season variations. An example query image and the corresponding rendered 3D model image are shown in figure 8.1.

For registration, an image-based method, as described in chapter 2 could have been used. To develop a method that is robust even in the presence of considerably more snow than in figure 8.1, the pose estimation method proposed in Paper A is based on registration of height information computed from images with a height map extracted from a DEM, i.e. a 3D-3D registration. 

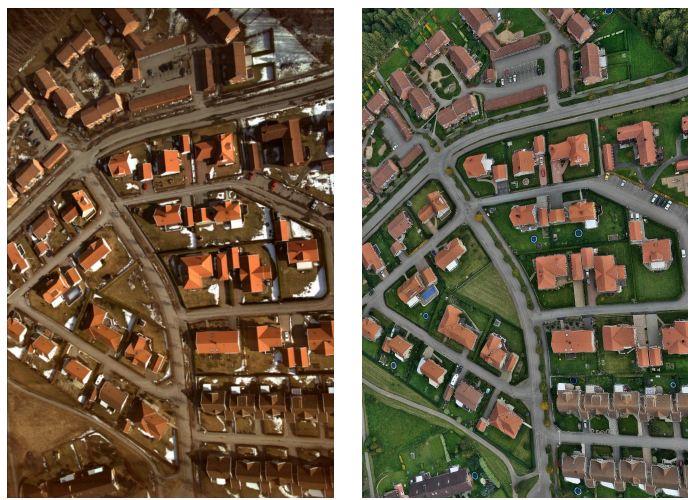

Figure 8.1: True (left) and rendered aerial image (right) from the same camera pose at different seasons. Images from Paper A.

A dense 3D reconstruction was generated from two consecutive images using Structure-from-Motion with the concept described in section 4.4. A rough global pose was assumed for camera 1 . The relative rotation between the camera poses was taken from IMU data and the relative translation direction was estimated from FAST image correspondences [46] and the epipolar constraint. The dense 3D reconstruction was then orthorectified based on the global pose assumption for camera 1 to generate a local height patch, i.e. the height $h_{\mathrm{p}}$ for the local area jointly seen in the two images. A height map $h_{\mathrm{m}}$ is extracted from the $3 \mathrm{D}$ model from, what is assumed to be, the corresponding area. Example images of the local height patch and the corresponding height map are shown in figure 8.2. The intensity in the images represents the height above the ground.
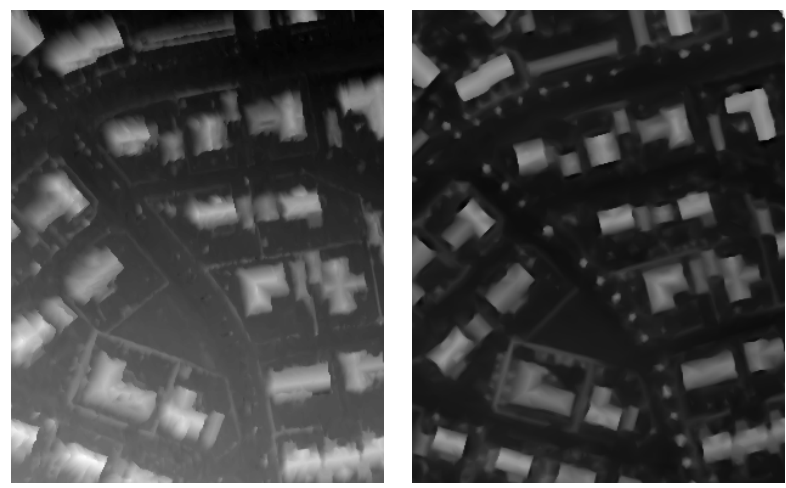

Figure 8.2: Local height patch (left) and 3D map (right) from the same suburban area. Images from Paper A. 
Due to the errors in the global pose assumption for camera 1, there will be a rotation $\mathbf{R}$ and a translation $\mathbf{t}$ between the height patch and the height map. Furthermore, the absolute scale $\mathrm{S}$ of the height patch is not accurately known. In total, the mapping between a $3 \mathrm{D}$ point in the height patch $\mathbf{x}_{\mathrm{p}}$ and the corresponding point in the height map $\mathbf{x}_{\mathrm{m}}$ is given by

$$
\left(\begin{array}{c}
x_{\mathrm{p}} \\
y_{\mathrm{p}} \\
z_{\mathrm{p}} \\
1
\end{array}\right)=\left(\begin{array}{cc}
\mathbf{S R} & \mathbf{t} \\
0 & 1
\end{array}\right)\left(\begin{array}{c}
x_{\mathrm{m}} \\
y_{\mathrm{m}} \\
z_{\mathrm{m}} \\
1
\end{array}\right)
$$

A detailed comparison of the height information in figure 8.2 shows that the height contour of the houses is more diffuse in the local height patch than in the height map. The effect is enhanced at remote house boundaries and is caused by stereo shadowing effects for the height patch which is based on two images only. This type of difference is not accounted for in (8.1) and is one error source for non-perfect registration.

The proposed solution in Paper A is to split the $3 \mathrm{D}$ registration into solving three 2D subproblems; first registration in the ground plane (XY), then in the two vertical planes $(\mathrm{XZ})$ and $(\mathrm{YZ})$. The justification for this approach is that the largest errors in our specific problem formulation, the XY position, the heading angle and the scale, are all considered in the ground plane, i.e. in the first $2 \mathrm{D}$ subproblem. The errors in the pitch and roll angles are expected to be considerably smaller, either using an estimate obtained from the methods in Papers B or C, or from an onboard IMU.

For alignment in the different planes we use an approach similar to the tracking part in [51]. We minimize the error function $\epsilon_{I J}$, the squared difference in height between the local height patch $h_{\mathrm{p}}(\mathbf{x})$ and the map $h_{\mathrm{m}}(\mathbf{x})$,

$$
\epsilon_{I J}=\int_{W}\left[h_{\mathrm{p}}(\mathbf{A} \mathbf{x}+\mathbf{d})-h_{\mathrm{m}}(\mathbf{x})\right]^{2} \omega(\mathbf{x}) d \mathbf{x},
$$

where $\mathbf{A}$ denotes a linear transformation, $\mathbf{x}$ is a point in the image, $\mathbf{d}=$ $\left[\begin{array}{ll}d_{\mathrm{x}} & d_{\mathrm{y}}\end{array}\right]^{T}$ is a displacement and $\omega$ is a window function. The indices $I J$ denote the three different planes. The integration domain $W$ is the whole patch area.

The error function in (8.2) is minimized by linearizing the integrand and differentiating with respect to all unknowns. In the XY-plane, the matrix $\mathbf{A}$ was a general affine transform whereas in the two vertical planes it was constrained to be a rotation matrix. The explicit error functions to be minimized in the different planes are given in Paper A.

From the alignment in the three different planes, an estimate of the rotation, translation and scale in (8.1) is obtained and from this information a refined estimate of the global pose can be computed. 


\section{Papers B and C - registration of 3D points on the unit sphere}

The global pose estimation method in Paper A requires a good initialization of the absolute pose of camera 1 to converge to a pose close to the true value. If the pitch and roll angles are known with high accuracy, the $3 \mathrm{D}$ registration problem in Paper A would collapse into a $2 \mathrm{D}$ registration problem in the ground plane. This argumentation was one of the drivers prior to developing the methods for attitude estimation in Papers B and C.

The method proposed in Paper B, described in section 6.1, uses edge detection and probabilistic Hough voting to estimate the aircraft attitude assuming a smooth spherical earth. The 3D-3D registration is here implicit in the process. We exploit the fact that for a smooth earth with no topography the horizon will generate a circular disc with radius $r(h)$ on the unit sphere. This is the connection between image content and the world reference frame that enables a global attitude estimate to be provided. The attitude errors using this method is from a few tenths of a degree up to one degree in an area with modest ground elevations, i.e. with elevations up to a few hundred meters. This is definitely acceptable as an initialization for the method in Paper A, but not accurate enough for the complete 6 DoF pose estimation to be considered a mere $2 \mathrm{D}$ registration problem.

In Paper C, all edge pixels within a thin band around the ideal horizon were extracted and projected onto the unit sphere. This point set is denoted $\mathbf{P}_{\mathrm{s}}$. If this point set is rotated with the transpose of the estimated camera rotation $\mathbf{R}_{\mathrm{c} \text {,est }}$, the new rotated point set on the sphere $\mathbf{P}_{\mathrm{r}}$ will ideally be the perceived horizon points rotated to a camera system aligned with the world coordinate system. Given an assumption of the aircraft global position and heading, the geometric horizon can be computed from a DEM and be projected onto the unit sphere. This point set from the geometric horizon is denoted $\mathbf{P}_{\text {geom }}$. We then have two point sets on the unit sphere, one originating from the image and one from the DEM. An example is shown in figure 8.3.

To refine the attitude estimate $\mathbf{R}_{c, \text { est }}$ that was obtained when assuming an ideal horizon, we search for a rotation such that the distance between the corresponding points in the two point sets is minimized. If we choose to compute the point set $\mathbf{P}_{\text {geom }}$ for the same angles $\alpha_{i}$ on the XY-plane as for the point set $\mathbf{P}_{\mathrm{r}}$, i.e.

$$
\alpha_{i}=\tan ^{-1}\left(\mathrm{P}_{\mathrm{r}_{\mathrm{y}}, i} / \mathrm{P}_{\mathrm{r}_{\mathrm{x}}, i}\right)
$$

the small arcs between the point sets will have their main component along the z-axis of the unit sphere. Using a small arc angle approximation, we define the error function to be the squared distance between the z-coordinate of the point sets, i.e.

$$
\epsilon_{z}=\sum_{i}\left(\mathrm{P}_{\mathrm{r}_{\mathrm{z}}, i}-\mathrm{P}_{\text {geom }_{\mathrm{z}}, i}\right)^{2}
$$



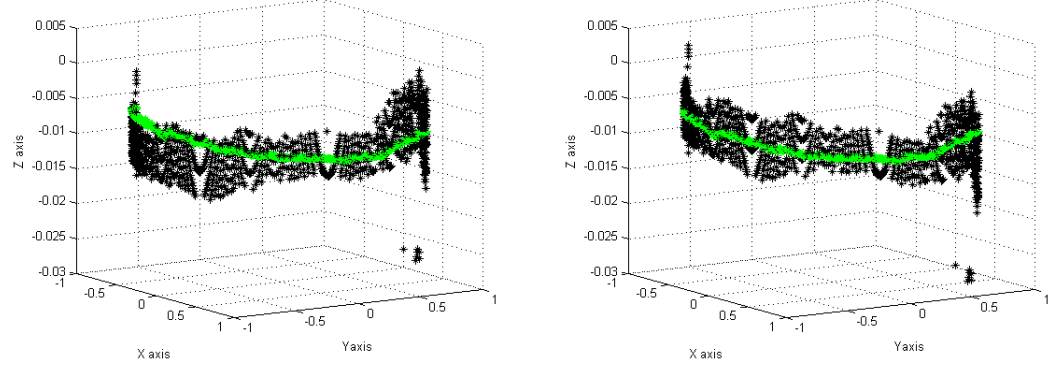

Figure 8.3: Horizon from image edge pixels (black) and from DEM (green), without refinement (left) and after refinement (right). Note the different scale on the $\mathrm{Z}$ axis. Images from Paper $\mathrm{C}$.

where the summation is made over all extracted horizon edge pixels. For attitude refinement, we search for the camera orientation $\mathbf{R}_{\mathrm{c}}$ that minimizes the error function in (8.4), i.e.

$$
\mathbf{R}_{\mathrm{c}, \mathrm{est}}=\arg \min _{\mathbf{R}_{\mathrm{c}}} \epsilon_{z}\left(\mathbf{R}_{\mathrm{c}}\right) .
$$

We minimize the nonlinear error function using the Levenberg-Marquardt algorithm [38] and initialize with the camera orientation matrix obtained from the Hough voting. Another conceivable solution to minimize the distance between the two point sets would be the orthogonal Procrustes method [50].

\section{$8.22 \mathrm{D}-2 \mathrm{D}$ registration}

\section{Papers E and F - spatial registration of horizon points on image plane}

In Papers $\mathrm{E}$ and $\mathrm{F}$, we want to register the horizon line in the image with the geometric horizon from the DEM to provide training labels for a CNN used for horizon line segmentation. The original panoramic image is warped to compensate for the camera orientation and to create an image corresponding to an approximately level camera, figure 8.4(a). A Canny detector is used to generate an edge image, figure $8.4(\mathrm{~b})$.

It is not obvious which edge pixels that originate from the horizon line, and which edges that are generated by clouds, objects on land, and the sea surface. A distance function $\mathrm{D}$ is computed from the edge image, where $\mathrm{D}$ is a measure of the distance to the nearest edge pixel. The geometric horizon line is computed for the position measured with the GPS and it is overlaid on the warped image, figure 8.4(c) and on the distance function 8.4(d). Ideally, the horizon line would be located where the distance function $\mathrm{D}$ is zero. For illustration purposes, the assumed heading angle has deliberately been set 


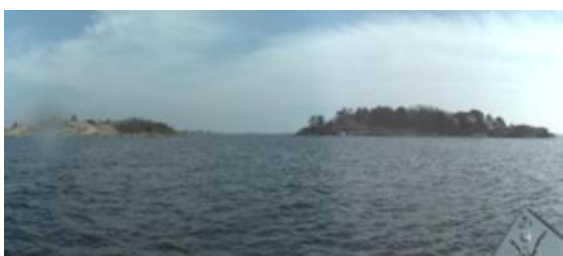

(a)

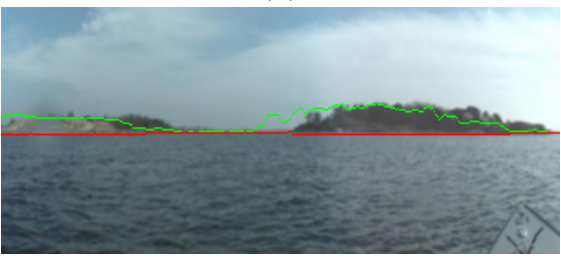

(c)

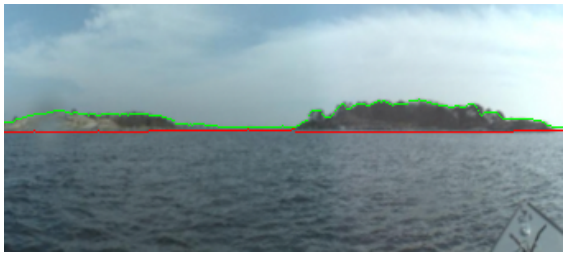

(e)

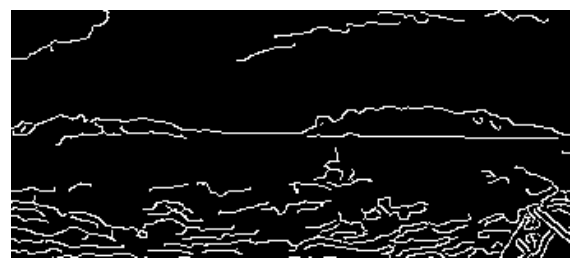

(b)

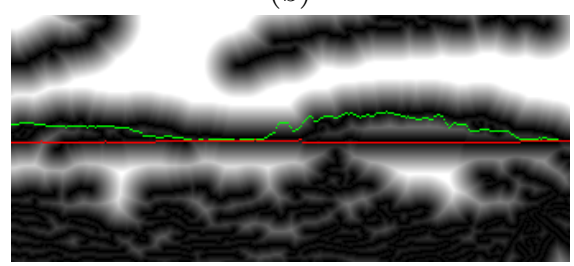

(d)

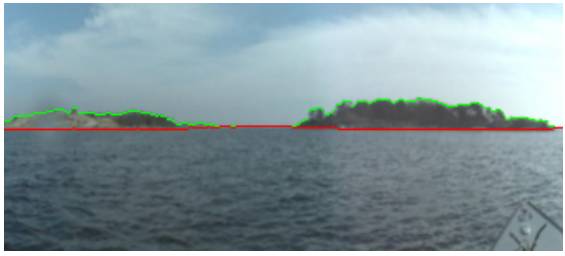

(f)

Figure 8.4: (a) Panoramic image warped with estimated orientation angles. (b) Edge map of warped image. (c) Geometric horizon (green) and water line (red) overlaid on image. (d) Distance function with geometric horizon overlaid. (e) Geometric horizon overlaid after orientation tuning. (f) Geometric horizon after subpixel tuning. Images from paper E.

slightly wrong in figures $8.4(\mathrm{c})$ and (d). This is the reason why the overlaid geometric horizon does not coincide with the horizon in the image and with the zeros of the distance function. For registration, we search for a rotation of the geometric horizon line points on the cylinder, such that when projected onto the image, their summed distance function values will be a minimum, i.e. we minimize the score

$$
s=\underset{\theta, \phi, \psi}{\operatorname{argmin}} \sum_{i} \mathrm{D}\left\{\mathbf{R}(\psi) \pi\left(\mathbf{R}(\theta, \phi) \pi^{-1}\left(h_{i}\right)\right)\right\}
$$

where $h_{i}$ are the horizon line points, $\pi$ is the projection from the cylinder to the image plane, $\theta, \phi$, and $\psi$ are the pitch, roll and heading angles, $\mathbf{R}$ is a rotation matrix and $\mathrm{D}$ is the distance function.

We perform a grid search over the pitch, roll and heading angles. For the first two angles we need to compute the rotation matrix and project the transformed points onto the image plane. The heading angle rotation simply 
corresponds to a horizontal shift on the image plane for the cylindrical projection. We extract the rotation angles for the minimum score and project the geometric horizon line and water line onto the warped image after transformation with the said rotation angles, figure 8.4(e).

In general, there is a good fit between the geometric horizon line with the image content, but there are some small deviations. The main reason is that the DEM is not a perfect representation of the real world. To adjust for these discrepancies we perform a final tuning step of the geometric horizon line in the image.

For final tuning we first convolve the warped image with a Sobel filter to enhance horizontal gradients. For each image column we search for a local maximum of the gradient, exceeding a threshold, in a small region close to the horizon line obtained in the registration step. If no gradient maximum is detected in the search region, the horizon line from the registration is retained. The resulting horizon line after the fine tuning step is shown in figure 8.4(f).

\section{$8.31 \mathrm{D}-1 \mathrm{D}$ registration}

\section{MOSSE filter registration of horizon line}

In Papers E and F, we use a CNN-based method to extract the horizon line from the omnidirectional panoramic images, see figure 8.5.

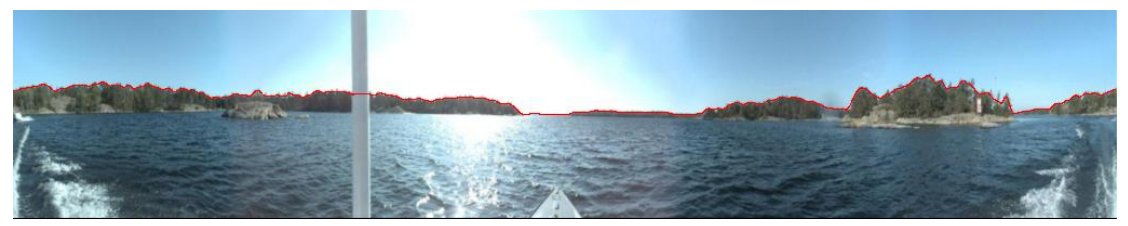

Figure 8.5: CNN based prediction of the horizon line (red) in Paper F.

For registration with the geometric horizon line, we adapt and employ the MOSSE correlation filter [5], originally developed for visual object tracking. The MOSSE filter is designed to generate a desired output signal (typically a Gaussian) shifted to the spatial location most closely corresponding to a set of learned reference signals. For efficiency, the filter is evaluated in the Fourier domain. The filter is trained with multiple references to improve its robustness against changes in appearance and noise.

In our case we use the segmented horizon lines from an image sequence as the reference signals. We align the segmented horizon lines in the spatial domain in accordance with their estimated heading angle. In the Fourier transform domain, we denote the horizon lines in the sequence with $F_{i}$ and 
the Gaussian target signal with $G$. We compute the MOSSE filter $K$ as

$$
K^{\star}=\frac{\sum_{i} G \cdot F_{i}^{\star}}{\sum_{i} F_{i} \cdot F_{i}^{\star}},
$$

where $\star$ denotes the complex conjugate and $\cdot$ element-wise multiplication. The MOSSE filter signal response is computed as

$$
r=\mathscr{F}^{-1}\left\{H \cdot K^{\star}\right\},
$$

where $H$ is the Fourier transform of the geometric horizon line from the DEM. Ideally, $r$ will be the Gaussian target signal with a zero phase shift if the heading angle estimate is correct. Examples of the MOSSE correlation filter signal response for a true and false position hypothesis are given in figure 8.6.

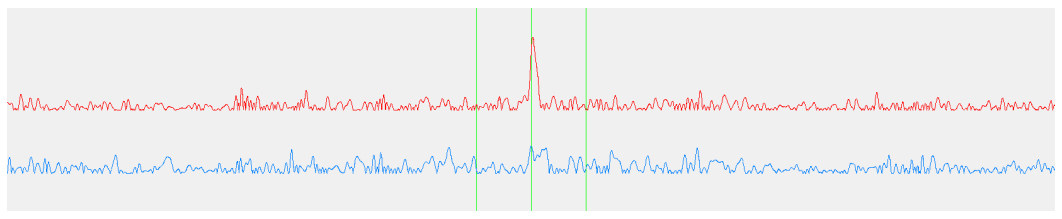

Figure 8.6: MOSSE correlation score assuming true (red) and false (blue) positions. The green lines indicate the center position and the search window.

Since the absolute heading could be slightly wrong, we search for the peak signal response within a small angular band from the center. As a quality measure of the signal response, i.e. our MOSSE filter correlation score, we use the peak-to-output-energy ratio [35]. We suppress the response within the expected Gauss signal bandwidth around the detected peak and compute the average energy over the remainder of the signal response. The correlation score is computed as the peak signal over the square root of the average energy.

To obtain a position estimate, we make a grid search and compute the MOSSE filter correlation score for various positions in a search area. The location of the maximum correlation score is extracted as the estimated position, and interpolation is used to achieve sub-grid position estimates.

\section{Method and component summary}

For the vision-based pose estimation methods, we now have methods to register image information with geometric geographic information to infer the global camera position and/or orientation. To determine the accuracy and robustness of the vision-based pose estimates, a comparison with a ground truth pose obtained with an independent sensor system is required. This comparison is made in the last system component called evaluation. 


\section{9}

\section{EvALUATION}

The aim of the methods presented in this thesis was to estimate the global position and/or attitude of the vehicle-mounted cameras. The most relevant error measure of the results hence is a comparison with the ground truth global position and attitude, if they are available. Another important aspect to evaluate is the robustness of the methods, i.e. at what rate do they generate accurate results. This chapter describes how the results of each method in the thesis were evaluated and how the ground truth was generated.

\subsection{Ground truth generation}

As mentioned in the introduction, the research work leading to this thesis has been conducted in two programs with close collaborations between industry and academia. One big advantage being an industrial $\mathrm{PhD}$ student employed at the aircraft manufacturer SAAB is the availability of true aerial images with accurate ground truth data. Having an accurate ground truth global 6 DoF pose for aerial imagery from outdoor large scale field trials is relatively rare in academia, which was evident when comparing the performance of the developed methods with other methods presented in the literature.

In Paper A, two large sets of aerial images from the Linköping area were used to evaluate the accuracy of the proposed method. Both sets of images had been processed by $\mathrm{SAAB}^{1}$ to reconstruct a $3 \mathrm{D}$ model of the area. The ground truth pose used for the individual images is the output from the bundle adjustment in this process. Evaluation of these 3D models has shown that the camera position accuracy is within a few centimeters and the attitude accuracy of the Euler angles (yaw, pitch and roll) is better than $0.01^{\circ}$.

In Paper $\mathrm{C}$, a dedicated flight trial with a fisheye camera was performed to capture the images required in order to evaluate the accuracy of the global

\footnotetext{
${ }^{1}$ The $3 \mathrm{D}$ reconstruction process is today owned by the SAAB spin-off company Vricon.
} 
vehicle attitude estimates. Onboard the aircraft, there was a GPS and a highly accurate IMU. A filtered GPS and IMU solution from the flight was computed by the navigation department at SAAB. The position accuracy is claimed to be a few centimeters and the attitude errors about $0.01^{\circ}$ for the pitch and roll angles and $0.04^{\circ}$ for the yaw angle.

In Papers E and F, the ground truth position of the USV carrying the panoramic camera was measured with a GPS onboard the USV. There was no inertial navigation system (INS) available onboard the USV, mainly due to payload size restrictions on the small USV. This means that the measured GPS positions could not be supported and refined by filtering with inertial measurements. The GPS position accuracy is estimated to be about $1.0-2.0 \mathrm{~m}$, but varies depending on the location on earth [59]. Without an INS, there was no ground truth available for the camera orientation measurements.

\subsection{Evaluation measures}

In Paper $\mathrm{A}$, the global $6 \mathrm{DoF}$ pose for a pair of images was estimated. The method is based on registration of a local height patch, computed with Structure-from-Motion, with a 3D model. The distance travelled for the camera between the two images is also unknown and estimated as a scale ratio between the local height patch and the $3 \mathrm{D}$ model. For the $6 \mathrm{DoF}$ pose, the deviation from the true global position and the deviation in the vehicle Euler angles (yaw, pitch and roll) was used as the error measure for the method. For the scale, i.e. the stereo baselength between the cameras, the relative error in percent was used as the evaluation measure. The horizontal position errors obtained were in the order of $1-2 \mathrm{~m}$ and the attitude errors were about $1-3 \mathrm{mrad}$. The ground truth was at least 10 times more accurate and hence sufficiently accurate for evaluation of the proposed method.

In Paper A, the registration rate as a function of the initialization errors was reported to compare the performance of our method with similar methods in the literature. The judgement whether a registration is considered correct or not is to some degree a subjective measure. Just by plotting the absolute 6 DoF pose errors, it was very evident which pose estimates that had been trapped in a local minimum in the optimization process and generated large errors. For a few cases, where the absolute orientation errors were in a grey zone, a visual inspection was made to judge if the height patch and the height map were well aligned. Figure 9.1 shows an example where the local height patch, after transformation with the estimated pose, is well registered with the height map.

In Paper B, aerial images from the internet were used to evaluate the proposed attitude estimation method via horizon detection. For these images there was no ground truth attitude and no camera calibration available. To evaluate the performance of the method, a comparison was made between the 

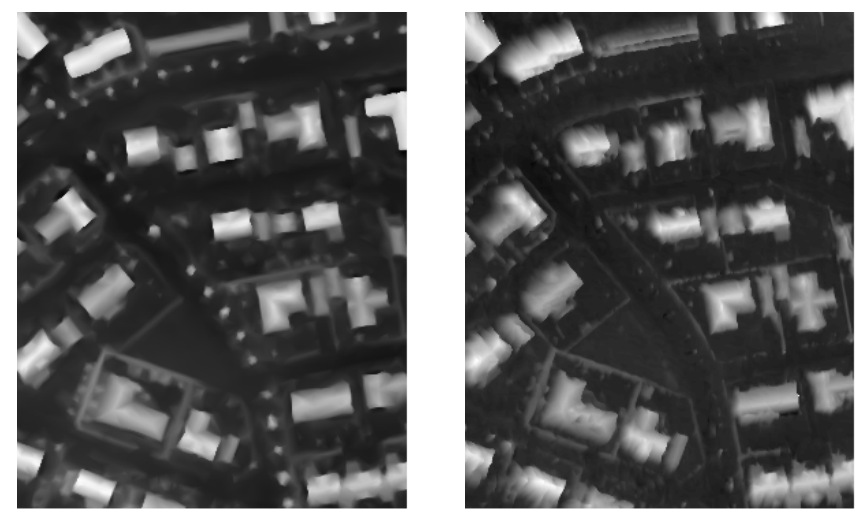

Figure 9.1: Height map from 3D model (left). Local height patch after transformation with estimated pose (right). Images are from Paper A.

manually estimated horizon on the image and the horizon estimate from the method. One image from Paper B is shown in figure 9.2 where the estimated horizon is overlaid on the image. The agreement with the true horizon line is good, but it is truly a subjective measure and the evaluation method is only suitable for small datasets.
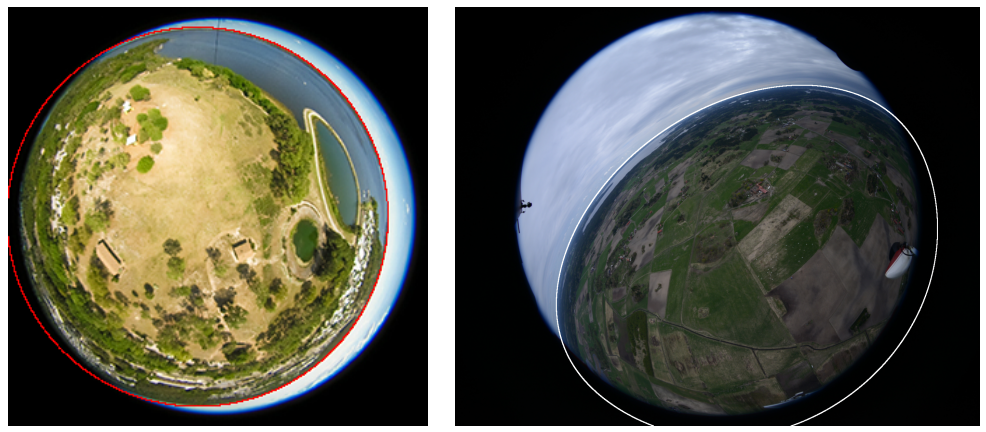

Figure 9.2: Images from Paper B (left) and Paper C (right) with the estimated horizon overlaid. The image from Paper B is from markmarano.com.

In Paper C, the absolute global pose of the aircraft was available from navigation sensors. Our method estimates the absolute pitch and roll angles via horizon detection and the evaluation measure is the deviation in the pitch and roll angles from the ground truth value. The attitude estimates obtained over 1600 images had mean errors less than $0.02^{\circ}$ and standard deviations less than $0.05^{\circ}$. These attitude estimates are truly highly accurate and the full potential of the method could not have been proven in the field trials without the GPS and INS-based ground truth which was about five times 
more accurate than the proposed method. The ground truth was computed through batch processing sensor data forward and backward over the entire flight and is no online method. The experiments also proved that without taking ray refraction into account, a systematic error on the total tilt angle of about $0.05^{\circ}$ was generated. This effect could not have been proven without a high quality ground truth. One image from Paper $\mathrm{C}$ is shown in figure 9.2 where the estimated horizon is overlaid on the image. The agreement with the true horizon line in the image is very good but an accurate evaluation requires a quantitative comparison with ground truth data.

In Papers E and F, the global position of the USV in the archipelago was estimated via horizon detection in the panoramic image and registration with a geo-referenced DEM. The ground truth position was measured with a GPS. The error measure used in Papers $\mathrm{E}$ and $\mathrm{F}$ was the difference between the position estimates from the proposed method and the GPS measurements. The mean position errors were found to be in the order $2.5-3.5 \mathrm{~m}$. An analysis in Paper F shows that if the mean error is $2.5 \mathrm{~m}$, the proposed method and the GPS measurements contribute more or less equally to the total error. The real mean error of the estimates from the true position would in that case be about $1.7 \mathrm{~m}$. This analysis illustrates that an accurate ground truth is essential to really evaluate the accuracy of your proposed method.

The position estimates from one of the trials in Paper $\mathrm{F}$ is shown in 9.3. The agreement with GPS measurements is very good, but on this scale the true accuracy cannot be determined. However, the figure indicates that the position accuracy is good enough for safe navigation of the USV in the archipelago. The missing data depends on frame drops in the data acquisition system and the horizon being outside the image border in the narrow straight.

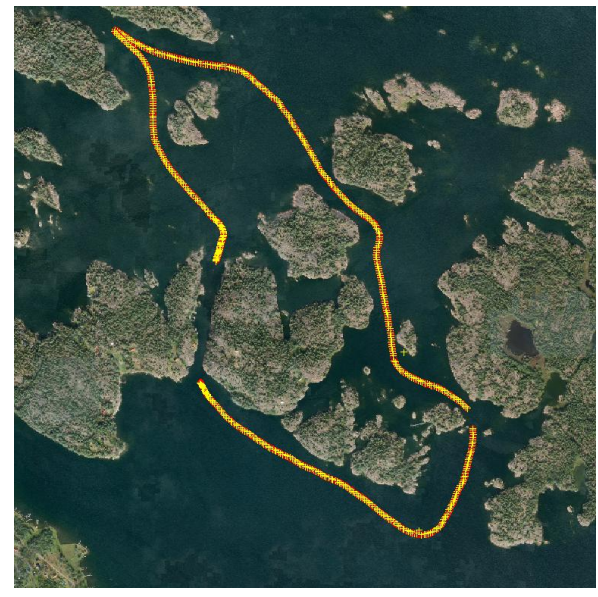

Figure 9.3: Test image position estimates with the GPS (red) and the proposed method in Paper F (yellow). 


\subsection{Evaluation analysis of onboard operational use}

In the previous section, the proposed pose estimation methods in this thesis were evaluated in terms of quantitative accuracy and robustness. The intention of this section is to analyze the potential and practical use of the methods in their respective operational environments onboard a $\mathrm{UxV}$.

\section{DoF pose estimates in Paper A}

The proposed method in Paper A estimates the 6 DoF pose of a UAV. The horizontal position errors obtained were in the order of $1-2 \mathrm{~m}$ and the attitude errors were about 1-3mrad. The accuracy of the pose estimates is definitely sufficient for navigation of a UAV during a normal mission. The main drawback of the method is the computational complexity. The computation time with the prototype implementation was around 20s per image pair, which is quite far from real time and practical onboard use. The method can be considered more a proof of concept. It shows that it is possible to accurately estimate the $6 \mathrm{DoF}$ pose of a UAV via registration of altitude information from images with a digital elevation model. To be operational for navigation onboard a UAV, the altitude registration must be made with a method that is significantly faster.

\section{Attitude estimates in Paper $\mathrm{C}$}

The proposed method in Paper $\mathrm{C}$ provides measurements of the absolute pitch and roll angles of a UAV. The attitude estimates obtained, over a one hour flight with 1600 images, had mean errors less than $0.02^{\circ}$ and standard deviations less than $0.05^{\circ}$. To judge the practical use of the method and the accuracy obtained, a comparison needs to be made with the performance of IMUs, the standard sensor for attitude measurements onboard a UAV. A thorough analysis of IMUs is outside the scope of this thesis, but some rule-ofthumb statements can be made. First of all, IMUs measure relative rotations and the estimates of absolute angles suffer from drift over time, the longer the mission the larger the absolute errors. Commercially available IMUs are divided into different classes based on their performance [53], see table 9.1. The gyroscope in-run bias in the table gives the order of magnitude of the

Table 9.1: Performance classes of IMUs.

\begin{tabular}{|l|c|}
\hline Class & Gyro in-run bias \\
\hline Automotive grade & $\sim 100^{\circ} / \mathrm{h}$ \\
Industrial grade & $\sim 10^{\circ} / \mathrm{h}$ \\
Tactical grade & $\sim 1^{\circ} / \mathrm{h}$ \\
Navigation grade & $\sim 0.1^{\circ} / \mathrm{h}$ \\
\hline
\end{tabular}


angular drift, and it can be used to give a rough estimate of absolute angular errors depending on the duration of a mission. As an example, after 6 minutes of operation, the absolute angular error of an industrial grade IMU is about $1^{\circ}$. The price range of IMUs varies from the order of 10 USD for an automotive grade IMU to the order of 100000 USD for a navigation grade IMU. Camera sensor systems are inexpensive in comparison with high-end IMUs.

Assuming that the UAV mission will last more than 10 minutes (a rather conservative assumption), the method in Paper $\mathrm{C}$ provides more accurate absolute attitude estimates than tactical grade IMUs. This means that the method will definitely aid and improve the orientation estimates in the navigation solution onboard the UAV. How this improvement in attitude measurement accuracy translates to operational efficiency of the UAV is a very complex task to analyze. It is application dependent and the task is left to the system design team of the UAV. The computation time with the prototype code is about a factor of three from real time, indicating that the method is real-time capable with some optimization. Based on this analysis, my statement on the operational use of the method in Paper $\mathrm{C}$ onboard a UAV is

- For missions longer than 10 minutes, the proposed method in Paper $\mathrm{C}$ provides robust absolute pitch and roll angle estimates with higher accuracy than a tactical grade IMU, and it improves the navigation solution onboard the UAV.

\section{Position estimates in Paper F}

The proposed method in Paper F achieved a position accuracy of about 2.5$3.5 \mathrm{~m}$ in two separate field trials. The position accuracy is the mean error computed over 1300 position estimates generated in the two trials. The numerical accuracy (GPS-level accurate) and the robustness of the position estimates, illustrated in figure 9.3, indicate that the method provides position estimates that are robust and accurate enough for safe navigation of a USV in the archipelago. The individual position estimates are based on images captured during a period of $1 \mathrm{~s}$. The prototype code runs at about $0.5 \mathrm{fps}$ and will readily run in real time after some optimization. There are some missing position estimates along the trajectory in the trials, but they can be explained by imperfections in the data acquisition system (frame drops) and hardware limitations (horizon line outside the vertical camera FOV). These types of failure cases will be handled in regular technical development of a sensor system and the system requirements placed on the USV. They are not limitations in the method per se. Based on this analysis, my statement on USV onboard operational use of the method in Paper F is

- The proposed method in Paper F generates robust position estimates with an accuracy that provides safe navigation of a USV operating in the archipelago. 


\section{0}

\section{CONCLUding REMARKS}

The aim of this thesis was to develop vision-based methods for global pose estimation of autonomous vehicles in order to aid their navigation. It is obvious that a multitude of methods will be required to handle the numerous combinations of environments (air, land, sea), terrain types (urban, rural), seasons, lighting conditions, and camera types in which the autonomous systems will operate. This thesis is mainly focused on methods to provide accurate pose estimates in natural environments, i.e. beyond urban areas, and using omnidirectional cameras. The amount of research conducted in this field is in my opinion surprisingly rare. With the total scope in mind, the methods and the results presented in this thesis have contributed with a few more pieces to solve the overall large jigsaw puzzle, vision-based methods for localization and attitude estimation to aid navigation of autonomous systems.

\subsection{Conclusions of results}

Paper A presents a method for global pose estimation in a suburban area assuming that the aerial vehicle is flying at a sufficient height above the houses and treetops to enable reconstruction of a local height map of the area. The local height map is registered with a georeferenced 3D model of the area to infer the global pose. The reason for matching altitude information is to make the method season invariant which is essential in the Swedish climate, where images of the same area may look very different depending on the season. The method in Paper A is more robust than any previously reported method on similar datasets in the sense that it provides the same, high level of correct registration rate for larger initialization errors than other methods. A limitation with the method is that it requires altitude structure in the scene.

The method for attitude estimation (pitch and roll angle) in Paper B is one of only a few methods that use an omnidirectional (fisheye) camera for horizon detection. It employs a probabilistic Hough voting scheme for horizon 
detection, which enables prior knowledge on the altitude and the pitch and roll angles to be exploited to make the attitude estimate more robust. Since the method in Paper B was evaluated on fisheye images from the internet with no ground truth pose and camera calibration available, no quantitative statements concerning the attitude accuracy could be made. However, the method proved promising enough to continue with the concept and perform our own flight trials with a fisheye camera to further evaluate the method.

The method in Paper $\mathrm{C}$ builds on the results and the method in Paper B. The additions made are unique and it is the very first time that an attitude estimate from horizon detection in fisheye images is refined through registration with the geometric horizon from a digital elevation model. In an aerial field trial, the mean and standard deviation of the pitch and roll angle errors are smaller than $0.02^{\circ}$ and $0.05^{\circ}$ respectively. To achieve the highly accurate attitude estimates, the ray refraction in the earth atmosphere was taken into account. This component is essential for highly accurate attitude estimates, but it has not been included in any previously reported attitude estimation method based on horizon detection.

The method in Paper $\mathrm{E}$ is the first paper where horizon detection and registration with elevation data is used to provide accurate position estimates in a marine environment. Two CNNs are designed and trained to estimate the camera orientation and to segment the horizon line in the images. The MOSSE correlation filter is adapted to horizon line registration with DEM data, and this method is shown to be more accurate and significantly faster than conventional registration in the spatial domain. Field trials conducted in the Swedish archipelago are used to demonstrate the GPS-level accuracy of the position estimates.

In Paper F, comprehensive field trials were conducted in the Swedish eastcoast archipelago over several days and in different regions to extend the method and the evaluation in Paper E. The results demonstrate that the proposed method can be trained on images captured in one area, and then applied to images from another, previously unvisited test area. The results also show that the method can be used in a coarse-to-fine scale fashion to reduce the search time, and that the position accuracy of the method degrades gracefully when narrowing the camera FOV.

To summarize the results in this thesis, were the goals of the two projects CIMSMAP and WASP defined in section 1.2 achieved? My answer is a definite "Yes". To relate to the pose estimation concepts defined in section 2; Do the proposed methods utilize the same techniques as we humans do for pose estimation? At a conceptual level, I say "Yes", mathematically, I say "No".

In CIMSMAP, we could estimate the pose of an aerial vehicle by successfully matching image content (local altitude maps and the horizon line) with elevation models, thus satisfying the project goal. In the WASP project, we could accurately localize a USV in the archipelago from onboard images, which fulfills the project goal to develop pose estimation methods for autonomous 
vehicles. In addition, the secondary project goal, to explore how deep learning methods could contribute to this type of research was also achieved through the usage of CNNs in submodules of the proposed method.

One reflection I have made is that the proposed methods for vision-based pose estimation are based on similar concepts as we humans use, but the accuracy obtained with the computer vision methods is by far superior to the accuracy we humans can achieve with our vision system. When flying, we humans are able to match the relative location between buildings in a bird'seye view (local altitude maps) with a map to tell roughly where we are located. We can also use the perceived horizon line to give a rough orientation estimate. When driving a boat in the archipelago, we can detect a few landmarks around us and triangulate our current position. The pose estimates we humans can provide in these situations are rather coarse. The main reasons that make the computer vision methods significantly more accurate are: (1) Well calibrated cameras for geometric interpretation of image information, (2) Mathematical tools to simultaneously aggregate image information over a larger field of view than the human vision system is capable to use, and (3) Capability to register image information with high resolution reference data.

With this reflection in mind, I am sure that there are more, yet unexplored concepts that we humans use for pose estimation that can be transferred to and be further developed in the computer vision domain.

\subsection{Future work}

As stated above, many vision-based pose estimation methods are required until vision can be robustly used throughout all phases of a mission for localization, attitude estimation and navigation of an autonomous vehicle. The use of omnidirectional images for this purpose is surprisingly rare in the works presented in the literature. My belief is that there is a huge, yet unexploited, potential for this type of imagery in the applications mentioned above. Thanks to the very large field of view, the registration will be more robust and the estimates more accurate compared to the often used perspective images with rather limited FOV. Just as an example, one part of the fisheye image viewing the horizon could be used for attitude estimation using the method in Paper C. Another part of the fisheye image, viewing the ground, could be used as in Paper A to estimate the full global 6 DoF pose, where the accurate attitude estimates considerably would speed up the convergence rate.

Today, most vision-based methods utilizing multiple modality data perform the registration of image information with elevation data in the spatial domain. The MOSSE filter registration showed to be significantly faster and more robust than conventional registration in the spatial domain. Employing a similar concept, i.e. transferring the registration to the Fourier domain, ought to have the potential to improve the robustness and accuracy of other already published works and ideas for vision-based pose estimation methods. 
One more observation, the full potential of the method proposed in Papers $\mathrm{E}$ and $\mathrm{F}$ could not have been demonstrated in a large scale field trial without having access to very accurate reference data. When large scale, high resolution elevation models will be made available to a larger community than it is today, I am sure that the extent of research on vision-based methods intended for natural environments will increase.

Finally, returning to the reflection in the previous section, there must still be more concepts that we humans use for pose estimation that can be transferred to and be further developed in the computer vision domain. Some conceivable concepts that we humans use are semantic reasoning and the combination of semantics in the scene with geometric information.

\subsection{Impact on society}

Technically, I see a large potential for current and future vision-based pose estimation methods being implemented onboard $\mathrm{UxVs}$ in various applications in natural environments. As was stated in section 9.3, the proposed methods in Papers $\mathrm{C}$ and $\mathrm{F}$ already provide technology that would be beneficial to the onboard navigation of $\mathrm{UxVs}$.

But new technology on its own is rarely the main driving force. Technology is an enabler, but there must be other incentives such as commercial interests, safety aspects or ethical reasons for technology to develop and spread abundantly. Today, almost everyone takes GPS for granted as the position sensor on a $\mathrm{UxV}$ operating in an outdoor environment. Adding a vision-based sensor system besides the GPS for localization implies an additional cost to the $\mathrm{UxV}$ system. In an operational cost analysis, it must be shown that this extra monetary cost can be compensated for by e.g. reduced operational costs (more accurate and efficient navigation solution), improved operational safety and robustness (fewer crashes and less damage due to more robust navigation), or enhanced ethical operational efficiency (less waste of medical supplies and food when more $\mathrm{UxVs}$ reach the target destination). It all boils down to what operational aspects and which parameters that have high weights in the overall $\mathrm{UxV}$ system cost function.

When the number of applications and the volume of $\mathrm{UxV}$ systems operating in natural environments in the future will reach some critical limit, I am confident that there will be $\mathrm{UxV}$ application segments where vision-based pose estimation sensors will be regarded as an equally important sensor as the GPS and IMU for robust onboard navigation. The analogy with the automotive industry is evident. Today, there is a segment of cars where vision sensors are compulsory for various driving assistance and safety systems. My strong belief is that the same phenomenon will occur within the world of UxVs. The day will come when there is a $\mathrm{UxV}$ segment where vision-based pose estimation sensors are considered mandatory for safe and robust operation of the $\mathrm{UxVs}$. 


\section{BIBLIOGRAPHY}

[1] R. Arandjelovic, P. Gronat, A. Torii, T. Pajdla, and J. Sivic. "NetVLAD: CNN architecture for weakly supervised place recognition". In: Proceedings of the IEEE Conference on Computer Vision and Pattern Recognition. 2016, pp. 5297-5307.

[2] G. Baatz, O. Saurer, K. Köser, and M. Pollefeys. "Large Scale Visual Geo-Localization of Images in Mountainous Terrain". In: ECCV (2012).

[3] D. H. Ballard. "Generalizing the Hough Transform to Detect Arbitrary Shapes". In: Pattern Recognition 13.2 (1981), pp. 111-122.

[4] G. Bao, Z. Zhou, S. Xiong, X. Lin, and X. Ye. "Towards Micro Air Vehicle Flight Autonomy Research on The Method of Horizon Extraction". In: $\operatorname{IMTC}(2003)$.

[5] D. S. Bolme, J. R. Beveridge, B. A. Draper, and Y. M. Lui. "Visual object tracking using adaptive correlation filters". In: Computer Vision and Pattern Recognition (CVPR), 2010 IEEE Conference on. IEEE. 2010, pp. 2544-2550.

[6] S. Bonadies, A. Lefcourt, and S. A. Gadsden. "A survey of unmanned ground vehicles with applications to agricultural and environmental sensing". In: Autonomous Air and Ground Sensing Systems for Agricultural Optimization and Phenotyping. Vol. 9866. International Society for Optics and Photonics. 2016, 98660Q.

[7] J. Brejcha and M. Čadík. "State-of-the-art in visual geo-localization". In: Pattern Analysis and Applications 20.3 (2017), pp. 613-637.

[8] J. Canny. "A computational approach to edge detection." In: PAMI 8 (1986), pp. 679-698.

[9] L.-C. Chen, G. Papandreou, I. Kokkinos, K. Murphy, and A. L. Yuille. "Deeplab: Semantic image segmentation with deep convolutional nets, atrous convolution, and fully connected crfs". In: IEEE transactions on pattern analysis and machine intelligence 40.4 (2018), pp. 834-848. 
[10] O. Chum, M. Perd'och, and J. Matas. "Geometric min-hashing: Finding a (thick) needle in a haystack". In: Computer Vision and Pattern Recognition, 2009. CVPR 2009. IEEE Conference on. IEEE. 2009, pp. 1724 .

[11] D.-A. Clevert, T. Unterthiner, and S. Hochreiter. "Fast and accurate deep network learning by exponential linear units (elus)". In: arXiv preprint arXiv:1511.07289 (2015).

[12] C. Demonceaux, P. Vasseur, and C. Pégard. "Omnidirectional vision on UAV for attitude computation". In: International Conference on Intelligent Robots and Systems (2006).

[13] J. Deng, W. Dong, R. Socher, L.-J. Li, K. Li, and L. Fei-Fei. "Imagenet: A large-scale hierarchical image database". In: Computer Vision and Pattern Recognition, 2009. CVPR 2009. IEEE Conference on. Ieee. 2009, pp. 248-255.

[14] S. J. Dumble and P. W. Gibbens. "Horizon Profile Detection for Attitude Estimation". In: Journal of Intelligent Robotic Systems 68 (2012), pp. 339-357.

[15] D. Dusha, L. Mejias, and R. Walker. "Fixed-Wing Attitude Estimation Using Temporal Tracking of the Horizon and Optical Flow". In: Journal of Field Robotics 28.3 (2011), pp. 355-372.

[16] M. Felsberg and G. Granlund. "P-channels: Robust multivariate mestimation of large datasets". In: Pattern Recognition, 2006. ICPR 2006. 18th International Conference on. Vol. 3. IEEE. 2006, pp. 262-267.

[17] M. Felsberg and J. Hedborg. "Real-time visual recognition of objects and scenes using p-channel matching". In: Scandinavian Conference on Image Analysis. Springer. 2007, pp. 908-917.

[18] https : / /www . wired . com/story/fukushima-robot-cleanup/. Accessed 2019-02-11.

[19] X. Glorot, A. Bordes, and Y. Bengio. "Deep sparse rectifier neural networks". In: Proceedings of the Fourteenth International Conference on Artificial Intelligence and Statistics. 2011, pp. 315-323.

[20] I. Goodfellow, Y. Bengio, and A. Courville. Deep learning. MIT press, 2016.

[21] G. Granlund and H. Knutsson. "Signal Processing for Computer Vision". In: Springer, ISBN 0-7929-9530-1 (1995).

[22] B. Grelsson, M. Felsberg, and F. Isaksson. "Global Pose Estimation of Aerial Images". In: $S S B A$ (2013).

[23] B. Grelsson. Global Pose Estimation from Aerial Images: Registration with Elevation Models. Licentiate thesis No. 1672. Linköping University Electronic Press, 2014. ISBN: 978-91-7519-279-6. 
[24] B. Grelsson and M. Felsberg. "Improved Learning in Convolutional Neural Networks with Shifted Exponential Linear Units (ShELUs)". In: 2018 24th International Conference on Pattern Recognition (ICPR). IEEE. 2018, pp. 517-522.

[25] B. Grelsson and M. Felsberg. "Probabilistic Hough voting for attitude estimation from aerial fisheye images". In: Scandinavian Conference on Image Analysis. Springer. 2013, pp. 478-488.

[26] B. Grelsson, M. Felsberg, and F. Isaksson. "Efficient 7D aerial pose estimation". In: Robot Vision (WORV), 2013 IEEE Workshop on. IEEE. 2013, pp. 88-95.

[27] B. Grelsson, M. Felsberg, and F. Isaksson. "Highly accurate attitude estimation via horizon detection". In: Journal of Field Robotics 33.7 (2016), pp. 967-993.

[28] B. Grelsson, A. Robinson, M. Felsberg, and F. Khan. "GPS-level Accurate Camera Localization with HorizonNet". In: Submitted to Journal of Field Robotics (2019).

[29] B. Grelsson, A. Robinson, M. Felsberg, and F. Khan. "HorizonNet for visual terrain navigation". In: 2018 3rd International Conference on Image Processing, Applications and Systems (IPAS). IEEE. 2018.

[30] M. Gyer. "Methods for Computing Photogrammetric Refraction Corrections for Vertical and Oblique Photographs". In: Photogrammetric Engineering and Remote Sensing 62.3 (1996), pp. 301-310.

[31] R. Hartley and A. Zisserman. "Multiple View Geometry in Computer Vision". In: Cambridge University Press, 2nd edition (2004).

[32] K. He, X. Zhang, S. Ren, and J. Sun. "Deep residual learning for image recognition". In: Proceedings of the IEEE conference on computer vision and pattern recognition. 2016, pp. 770-778.

[33] P. Hough. "Method and means for recognizing complex patterns." In: U.S. Patent 3069654 (1962).

[34] A. Irschara, C. Zach, J.-M. Frahm, and H. Bischof. "From structurefrom-motion point clouds to fast location recognition". In: Computer Vision and Pattern Recognition, 2009. CVPR 2009. IEEE Conference on. IEEE. 2009, pp. 2599-2606.

[35] B. Javidi and J. Wang. "Design of filters to detect a noisy target in nonoverlapping background noise". In: JOSA A 11.10 (1994), pp. 26042612 .

[36] A. Kendall, M. Grimes, and R. Cipolla. "Posenet: A convolutional network for real-time 6-dof camera relocalization". In: Computer Vision (ICCV), 2015 IEEE International Conference on. IEEE. 2015, pp. 29382946. 
[37] A. Krizhevsky, I. Sutskever, and G. E. Hinton. "Imagenet classification with deep convolutional neural networks". In: Advances in neural information processing systems. 2012, pp. 1097-1105.

[38] K. Levenberg. "A method for the solution of certain problems in least squares". In: Quarterly of applied mathematics 2 (1944), pp. 164-168.

[39] Y. Li, N. Snavely, D. Huttenlocher, and P. Fua. "Worldwide pose estimation using 3d point clouds". In: European conference on computer vision. Springer. 2012, pp. 15-29.

[40] T. G. McGee, R. Sengupta, and K. Hedrick. "Obstacle Detection for Small Autonomous Aircraft Using Sky Segmentation". In: ICRA (2005).

[41] C. Mei and P. Rives. "Single View Point Omnidirectional Camera Calibration from Planar Grids". In: IEEE International Conference on Robotics and Automation (2007).

[42] SRTM. http://www2.jpl.nasa.gov/srtm/. Accessed 2019-02-11.

[43] D. Nister. "An efficient solution to the five-point relative pose problem". In: Proc. Computer Vision and Pattern Recognition (2003), pp. 195-202.

[44] OceanAlpha. http://www. oceanalpha.com/. Accessed 2019-02-11.

[45] J. Redmon, S. Divvala, R. Girshick, and A. Farhadi. "You only look once: Unified, real-time object detection". In: Proceedings of the IEEE conference on computer vision and pattern recognition. 2016, pp. 779 788 .

[46] E. Rosten and T. Drummond. "Machine learning for high-speed corner detection". In: $\operatorname{ECCV}(2006)$.

[47] T. Sattler, B. Leibe, and L. Kobbelt. "Fast image-based localization using direct 2d-to-3d matching". In: Computer Vision (ICCV), 2011 IEEE International Conference on. IEEE. 2011, pp. 667-674.

[48] T. Sattler, A. Torii, J. Sivic, M. Pollefeys, H. Taira, M. Okutomi, and T. Pajdla. "Are Large-Scale 3D Models Really Necessary for Accurate Visual Localization?" In: 2017 IEEE Conference on Computer Vision and Pattern Recognition (CVPR). IEEE. 2017, pp. 6175-6184.

[49] D. Scaramuzza, A. Martinelli, and R. Siegwart. "A toolbox for Easily Calibrating Omnidirectional Cameras". In: International Conference on Intelligent Robots and Systems (2006).

[50] P. H. Schönemann. "A generalized solution of the orthogonal Procrustes problem". In: Psychometrika 31.1 (1966), pp. 1-10.

[51] J. Shi and C. Tomasi. "Good features to track". In: CVPR (1994).

[52] S. Thurrowgood, D. Soccol, R. J. D. Moore, D. Bland, and M. V. Srinivasan. "A Vision Based System for Attitude Estimation of UAVs". In: International Conference on Intelligent Robots and Systems (2009). 
[53] VectorNav. https : / / www . vectornav . com / support / library / imuand-ins. Accessed 2019-02-11.

[54] R. Verbickas and A. Whitehead. "Sky and ground detection using convolutional neural networks". In: Proceedings of the International Conference on Machine Vision and Machine Learning. Vol. 64. 2014.

[55] Vricon. http://www.vricon.com. Accessed 2019-02-11.

[56] N. J. Wade and S. Finger. "The eye as an optical instrument: from camera obscura to Helmholtz's perspective". In: Perception 30.10 (2001), pp. 1157-1177.

[57] https : / / liu . se / nyhet / har - testas - autonom - sjoraddning i - praktiken ? \&utm _ source = LiU - nytt \& utm_medium = email \& utm _ campaign=vecka-41-2018. Accessed 2019-02-11.

[58] T. Weyand, I. Kostrikov, and J. Philbin. "Planet-photo geolocation with convolutional neural networks". In: European Conference on Computer Vision. Springer. 2016, pp. 37-55.

[59] William J. Hughes Technical Center. "Global Positioning System (GPS) Standard Positioning Service (SPS) Performance Analysis Report." In: Report No. 86 (July 31, 2014).

[60] Y. Wu, F. Tang, and H. Li. "Image-based camera localization: an overview". In: Visual Computing for Industry, Biomedicine, and Art 1.1 (2018), p. 8.

[61] C. Yuan, Y. Zhang, and Z. Liu. "A survey on technologies for automatic forest fire monitoring, detection, and fighting using unmanned aerial vehicles and remote sensing techniques". In: Canadian journal of forest research 45.7 (2015), pp. 783-792.

[62] Z. Zhang. "A flexible new technique for camera calibration". In: IEEE Transactions on Pattern Analysis and Machine Intelligence 22.11 (2000), pp. 1330-1334.

[63] Zipline. http://www.flyzipline.com/. Accessed 2019-02-11. 



\section{PART II}

\section{Publications}

The papers associated with this thesis have been removed for copyright reasons. For more details about these see:

http://urn.kb.se/resolve?urn=urn:nbn:se:liu:diva-154159 


\section{PAPER A}

\section{Efficient 7D AERial Pose EstimATION}

B. Grelsson, M. Felsberg, and F. Isaksson. "Efficient 7D aerial pose estimation". In: Robot Vision (WORV), 2013 IEEE Workshop on. IEEE. 2013, pp. 88-95

(C) 2013 IEEE. Personal use of this material is permitted. Permission from IEEE must be obtained for all other uses, in any current or future media, including reprinting/republishing this material for advertising or promotional purposes, creating new collective works, for resale or redistribution to servers or lists, or reuse of any copyrighted component of this work in other works. 


\section{PAPER B}

\section{Probabilistic Hough Voting FOR ATTITUDE ESTIMATION FROM AERIAL FISHEYE IMAGES}

B. Grelsson and M. Felsberg. "Probabilistic Hough voting for attitude estimation from aerial fisheye images". In: Scandinavian Conference on Image Analysis. Springer. 2013, pp. 478-488

Reprinted/adapted by permission from Springer Nature: Springer Verlag Berlin Heidelberg. Scandinavian Conference on Image Analysis by JoniKristian Kämäräinen and Markus Koskela (c) 2013 


\section{PAPER C}

\section{Highly AcCuRAte Attitude Estimation VIA HORIZON DETECTION}

B. Grelsson, M. Felsberg, and F. Isaksson. "Highly accurate attitude estimation via horizon detection". In: Journal of Field Robotics 33.7 (2016), pp. $967-993$

(C) 2015 Wiley Periodicals, Inc. Personal use of this material is permitted. No part of the Wiley Materials may be copied, modified, adapted, translated, reproduced, transferred or distributed, in any form or by any means, and no derivative works may be made based on the Wiley Materials without the prior permission of Wiley Periodicals, Inc. 


\section{PAPER D}

\section{IMPROVED LEARNING IN \\ Convolutional Neural \\ NeTWORKS WITH SHIFTED \\ EXPONENTIAL LINEAR UNITS \\ (SHELUs)}

B. Grelsson and M. Felsberg. "Improved Learning in Convolutional Neural Networks with Shifted Exponential Linear Units (ShELUs)". In: 2018 24th International Conference on Pattern Recognition (ICPR). IEEE. 2018, pp. $517-522$

(C) 2018 IEEE. Personal use of this material is permitted. Permission from IEEE must be obtained for all other uses, in any current or future media, including reprinting/republishing this material for advertising or promotional purposes, creating new collective works, for resale or redistribution to servers or lists, or reuse of any copyrighted component of this work in other works. 


\section{PAPER E}

\section{HORIZONNET FOR VISUAL TERRAIN NAVIGATION}

B. Grelsson, A. Robinson, M. Felsberg, and F. Khan. "HorizonNet for visual terrain navigation". In: 2018 3rd International Conference on Image Processing, Applications and Systems (IPAS). IEEE. 2018

(C) 2018 IEEE. Personal use of this material is permitted. Permission from IEEE must be obtained for all other uses, in any current or future media, including reprinting/republishing this material for advertising or promotional purposes, creating new collective works, for resale or redistribution to servers or lists, or reuse of any copyrighted component of this work in other works. 


\section{PAPER F}

\section{GPS-LEVEL ACCurate CAmera LOCALIZATION WITH HORIZONNET}

B. Grelsson, A. Robinson, M. Felsberg, and F. Khan. "GPS-level Accurate Camera Localization with HorizonNet". In: Submitted to Journal of Field Robotics (2019) 


\section{FACULTY OF SCIENCE AND ENGINEERING}

Linköping Studies in Science and Technology, Dissertations No. 1977, 2019 Department of Electrical Engineering

Linköping University

SE-581 83 Linköping, Sweden

www.liu.se

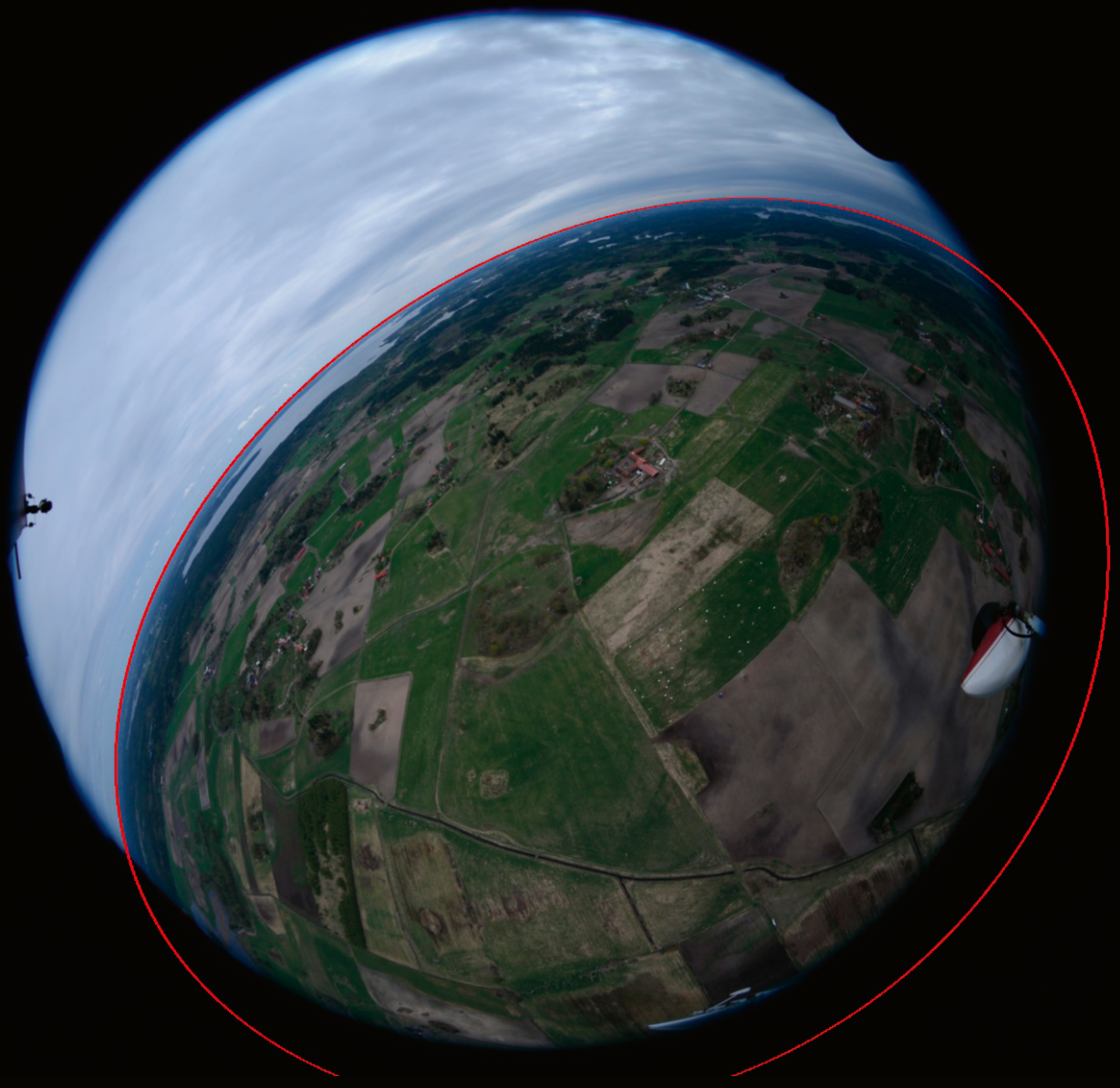

